\title{
ALGORITMO DE CONTROLE DE POTÊNCIA DISTRIBUÍDO FUNDAMENTADO NO MODELO POPULACIONAL DE VERHULST
}

\author{
Tadeu Júnior Gross, Taufik Abrão, Paul Jean Etienne Jeszensky
}

\begin{abstract}
Resumo - Neste artigo utiliza-se o modelo dinâmico contínuo de Verhulst, que foi elaborado inicialmente para descrever o crescimento populacional de espécies biológicas com restrição de alimento e espaço físico, para sintetizar um novo algoritmo de controle de potência distribuído (DPCA, Distributed Power Control Algorithm) aplicável em sistemas de múltiplo acesso por divisão de código (DS-CDMA, Direct Sequence-Code Division Multiple Access). Realiza-se a discretização da equação diferencial correspondente através da aplicação do método de Euler de integração numérica (NIE, Numerical Integration Euler Method). Estabelecem-se analiticamente as condições de convergência do DPCA proposto. As propriedades do algoritmo recursivo assim obtido, como distância Euclidiana do vetor ótimo em regime permanente, velocidade de convergência, erro quadrático médio normalizado (NSE, Mean Normalized Squared Error) na condição de regime permanente, consumo médio de potência por usuário e complexidade de implementação são investigados via simulações. Os resultados são confrontados com simulações análogas para o algoritmo clássico de Foschini e Miljanic e o sigmoidal de Uykan e Koivo. Na presença de erros de estimação, o DPCA proposto apresentou menor discrepância em regime permanente do vetor ótimo e maior velocidade de convergência. Adicionalmente, aplica-se a teoria dos Círculos de Gerschgorin (GC) na determinação de condições suficientes para que o problema de alocação seja factível.
\end{abstract}

Palavras-chave: DS-CDMA, DPCA, Modelo Populacional de Verhulst, Círculos de Gerschgorin.

\begin{abstract}
In this paper the continuous dynamic model of Verhulst is used. It had been elaborated initially to describe the population growth of biological species with food and physical space restrictions, in order to synthesize a new Distributed Power Control Algorithm (DPCA) applicable in Direct Sequence Code Division Multiple Access (DSCDMA) systems. The discretization of the corresponding differential equation is accomplished via Numeric Integration Euler method (NIE). Analytical convergence conditions for the proposed DPCA were also established. The properties of the proposed recursive algorithm, as Euclidian distance from optimum vector after convergence, convergence speed, Normalized mean Squared Error (NSE),
\end{abstract}

Tadeu Júnior Gross, Engenheiro Eletricista da Área Instrumental da Secretaria de Administração do Estado do Mato Grosso (SADMT), Cuiabá, MT, Brasil; Professor das Faculdades Unicen de Tangará da Serra, MT, Brasil. E-mail: tj.gross@uol.com.br Taufik Abrão, Universidade Estadual de Londrina, Departamento de Engenharia Elétrica, Paraná, Brasil. Email: taufik@uel.br Paul Jean Etienne Jeszensky, Escola Politécnica da Universidade de São Paulo, Departamento de Engenharia de Telecomunicações e Controle, São Paulo, Brasil. E-mail: pjj@ lcs.poli.usp.br average power consumption per user and implementation complexity are investigated through simulations. The simulation results are confronted with two another DPCA: the classic algorithm derived by Foschini and Miljanic and the sigmoidal of Uykan and Koivo. With estimation errors the proposed DPCA showed smaller discrepancy from the optimum power vector allocation after convergence and better convergence. Additionally, the Gerschgorin Circles theory (GC) is applied for the feasibility of the power allocation problem.

Keywords: DS-CDMA, DPCA, Verhulst Populational Model, Gerschgorin Circles.

\section{INTRODUÇÃO}

O trabalho de Foschini e Miljanic [1] constitui o alicerce de diversos DPCA's difundidos na literatura na medida em que o processo de síntese destes orienta-se no sentido de satisfazer à ODE (Ordinary Differential Equation) proposta em [1, Eq. (1)]; a diretriz da maior parte das investigações existentes na literatura sobre controle de potência distribuído tem sido a de aprimorar continuamente estruturas de controle atendo-se ao mesmo modelo dinâmico. Assim, a proposição de uma nova equação diferencial pode conduzir a algoritmos que sejam promissores sob algum aspecto significativo, tais como velocidade de convergência, proximidade do valor ótimo, sensibilidade a erros de estimação etc. Esse contexto justifica o novo modelo diferencial para síntese de DPCA's baseado na equação de Verhulst [2], proposto e analisado aqui.

Inicialmente, na Seção 2 descreve-se de forma sucinta o problema de alocação ótima de potência e deriva-se na Seção 3 um método, à luz da Teoria dos Círculos de Gerschgorin (GC) [3], que permite certificar de forma simples a existência do vetor ótimo. A seguir, na Seção 4 apresentam-se duas metodologias distribuídas, os DPCA's clássico [1] e sigmoidal [4], visando a resolução do problema do controle de potência em sistemas de comunicação de múltiplo acesso. A Seção 5 apresenta um novo DPCA baseado na ODE de Verhulst e na Seção 6 exploram-se as características de convergência do novo DPCA. Na Seção 7 são apresentados resultados numéricos comparativos entre os três algoritmos. Finalmente, a Seção 8 sintetiza as principais conclusões deste trabalho.

\section{O PROBLEMA DE ALOCAÇÃO DE POTÊNCIA ÓTIMA}

Em um sistema DS/CDMA multicelular com $K$ usuários móveis compartilhando o canal reverso, o sinal proveniente da unidade móvel $i$ será corretamente interpretado, com uma probabilidade de erro satisfatória, se a relação sinal - 
interferência mais ruído, SINR (Signal to Interference plus Noise Ratio) na estação rádio-base, ERB (Estação Rádio Base) não for inferior a um determinado valor, denotado por $\gamma_{i}^{*}$, estabelecido a priori. Portanto, deve-se controlar a potência transmitida de cada usuário móvel, $p_{i}$, com o intuito de obedecer a relação:

$$
\gamma_{i}=\frac{g_{i i} p_{i}}{\sum_{j=1, j \neq i}^{K} g_{i j} p_{j}+\sigma_{i}^{2}} \geq \gamma_{i}^{*}, i=1, \mathrm{~L}, K,
$$

onde $g_{i j}$ corresponde ao fator de atenuação do sinal do usuário interferente $j$ e $\sigma_{i}^{2}$ à potência do ruído AWGN (Additive White Gaussian Noise) inerente ao sistema de comunicação.

$\mathrm{O}$ vetor de potências que transforma as $K$ inequações acima em igualdades também minimiza a soma das potências transmitidas [5]. Portanto, impondo-se esta restrição, tem-se $K$ equações que podem ser convenientemente agrupadas em um formato matricial:

$(\mathbf{I}-\mathbf{H}) \mathbf{p}=\boldsymbol{\eta}$,

onde $h_{i j}=\gamma_{i}^{*} g_{i j} g_{i i}^{-1}, h_{i i}=0, \eta_{i}=\gamma_{i}^{*} \sigma_{i}^{2} g_{i i}^{-1}$ e $\mathbf{p}=\left[p_{1} \mathrm{~K} p_{K}\right]^{T}$.

A síntese de um DPCA consiste em desenvolver um procedimento sistemático de evolução do vetor p, baseando-se nos valores $\gamma_{i}^{*}, \gamma_{i}$ e $p_{i}$, para o vetor ótimo:

$\mathbf{p}^{*}=(\mathbf{I}-\mathbf{H})^{-1} \boldsymbol{\eta}$

A existência de uma solução não negativa para (2) segue de dois teoremas, atribuídos a Perron e Frobenius [6], sobre autovalores de matrizes não negativas.

Teorema 1: Se $\boldsymbol{S}$ é uma matriz quadrada não negativa, existe um autovalor $\rho$, denominado autovalor de PerronFrobenius, de forma que: 1) $\rho$ é real e não negativo; 2) $\rho \geq|\lambda|$ para qualquer autovalor $\lambda$ de $\boldsymbol{S}$. No plano complexo, o raio $\mathrm{r}(\mathbf{S})$ do menor círculo que contém todos os autovalores de uma matriz $\mathbf{S}$ qualquer, ou seja, $\mathrm{r}(\mathbf{S})=\max \{|\lambda|: \lambda \in \lambda(\mathbf{S})\}$, denomina-se raio espectral da matriz S [3]. Portanto, para uma matriz quadrada não negativa, raio espectral e autovalor de Perron-Frobenius podem ser utilizados sem distinção.

Teorema 2: Para uma matriz não negativa irredutível $\mathbf{S}$ e um vetor c estritamente positivo, $\mathbf{x}=\mathbf{S x}+\mathbf{c}$ possui solução $\mathbf{x}$ não negativa se, e somente se, o autovalor $\rho$ de PerronFrobenius for menor que a unidade.

Da equação (2) e do Teorema 2, conclui-se que o sistema em questão é factível se o raio espectral da matriz $\mathbf{H}$ for $\rho_{\mathbf{H}}$ $<$ 1. Portanto, torna-se necessário identificar quais condições os elementos da matriz $\mathbf{H}$ devem satisfazer de forma que os autovalores estejam limitados ao círculo unitário.

Uma maneira precisa de relacionar os elementos de $\mathbf{H}$ com seus autovalores consiste em analisar a influência dos coeficientes do polinômio característico sobre suas raízes. Este procedimento clássico torna-se inadequado quando o grau do polinômio for $n>4$, pois, exceto em casos particulares, não existe solução fechada para encontrar zeros de tais polinômios. Neste contexto, métodos aproximados para localizar autovalores revelam-se importantes.
A Teoria dos GC [3] permite a obtenção de um procedimento simples para se delimitar regiões do plano complexo que contêm autovalores. A utilização desta teoria na derivação de condições suficientes para garantir viabilidade constitui procedimento não explorado na literatura referente a controle de potência e será descrito a seguir.

\section{SISTEMA FACTÍVEL E OS ELEMENTOS DA MATRIZ H À LUZ DA TEORIA DOS CÍRCULOS DE GERSCHGORIN}

$\mathrm{O}$ Teorema de GC aliado às características particulares da matriz $\mathbf{H}$ revela-se uma ferramenta prática na obtenção de condições suficientes para viabilidade de um sistema de controle de potência. O Teorema de GC pode ser enunciado como [3]:

Teorema 3: $\mathrm{O}$ espectro $\lambda(\Delta)$ de uma matriz $\Delta_{n \times n}$ está contido em uma união $G(\Delta)$ de círculos centrados nos elementos da diagonal principal. $\mathrm{O}$ raio $R_{i}(\Delta)$ da região circular $\mathscr{D}_{i}(\Delta)$ associada a $\delta_{i i}$ é obtido somando-se os módulos dos elementos pertencentes à linha $i$ que não estejam localizados na diagonal principal, ou seja:

$$
\begin{aligned}
& R_{i}(\Delta)=\sum_{1=1,1 \neq i}^{n}\left|\delta_{i 1}\right| \\
& \mathcal{D}_{i}(\Delta)=\left\{\mathrm{z} \in C:\left|\mathrm{z}-\delta_{i i}\right| \leq R_{i}(\Delta)\right\} \\
& G(\Delta)=\bigcup_{i=1}^{n} \mathcal{D}_{i}(\Delta) \\
& \lambda(\Delta) \subset G(\Delta)
\end{aligned}
$$

Os discos $\mathscr{D}_{i}(\Delta), i=1, \ldots, n$, são denominados Círculos de Gerschgorin (GC) [3] e o fato de os autovalores de $\Delta$ estarem localizados na união dessas regiões pode ser referenciado como Teorema de GC. Cada disco disjunto necessariamente contém um autovalor.

Os espectros de matrizes transpostas são idênticos, e, portanto, $\lambda(\Delta) \subset G(\Delta) \cap G\left(\Delta^{\mathrm{T}}\right)$. Esta intersecção diminui a região de busca no plano complexo, melhorando a estimativa inicial sobre a localização dos autovalores.

Os elementos da diagonal principal da matriz $\mathbf{H}_{n \times n}$ são nulos por definição e, portanto, todos os GC são concêntricos e centrados na origem do plano complexo. Desta forma, o disco $\mathscr{D}_{k}(\mathbf{H})$ de maior raio contém a união de todos os GC, ou seja, existe pelo menos um $k \in\{1, \ldots, n\}$ de modo que $G(\mathbf{H}) \subset \mathscr{D}_{k}(\mathbf{H})$. Conseqüentemente, para este índice $k$ vale a desigualdade $\mathrm{r}(\mathbf{H}) \leq R_{k}(\mathbf{H})$. Analogamente, como $\lambda(\mathbf{H})=\lambda\left(\mathbf{H}^{\mathbf{T}}\right)$, obtém-se $\mathrm{r}(\mathbf{H}) \leq R_{\lambda}\left(\mathbf{H}^{\mathbf{T}}\right)$ para algum $\lambda \in\{1, \ldots, n\}$. Agrupando-se estas duas desigualdades obtémse um limite mais estreito para o raio espectral, ou seja, $\mathrm{r}(\mathbf{H}) \leq \min \left\{R_{k}(\mathbf{H}), R_{\lambda}\left(\mathbf{H}^{\mathbf{T}}\right)\right\}$.

A não negatividade da matriz quadrada $\mathbf{H}$ implica que seu raio espectral $r(\mathbf{H})$ e seu autovalor de Perron-Frobenius $\rho_{\mathbf{H}}$ são iguais e, portanto, $\rho_{\mathbf{H}} \leq \min \left\{R_{k}(\mathbf{H}), R_{\lambda}\left(\mathbf{H}^{\mathbf{T}}\right)\right\}$. Como um sistema factível deve necessariamente possuir $\rho_{\mathbf{H}}<1$, a restrição $\min \left\{R_{k}(\mathbf{H}), R_{\lambda}\left(\mathbf{H}^{\mathbf{T}}\right)\right\}<1$, onde $k$ identifica o maior 
disco da matriz $\mathbf{H}$ e $\lambda$ o maior de sua transposta, estabelece uma condição suficiente para viabilidade.

Sistematicamente, os passos para verificar se o problema de alocação de potências descrito por (2) tem solução ótima são:

1- Calcular a soma dos elementos da linha de $\mathbf{H}$ que possuir maior norma:

$$
R_{\text {row }}^{\text {max }}=\max _{k}\left\{\sum_{1=1}^{n} h_{k 1}\right\}
$$

2- Calcular a soma dos elementos da coluna de $\mathbf{H}$ que possuir maior norma:

$$
R_{c o l}^{\max }=\max _{1}\left\{\sum_{k=1}^{n} h_{k 1}\right\}
$$

3- Excluir o maior valor entre os dois anteriores:

$$
R_{G_{r} \cap G_{c}}^{\max }=\min \left\{R_{\text {row }}^{\max }, R_{c o l}^{\max }\right\}
$$

4- Valor resultante menor do que 1 implica viabilidade:

$R_{G_{r} \cap G_{c}}^{\max }<1 \Rightarrow$ sistema factível

$R_{G_{r} \cap G_{c}}^{\max } \geq 1 \Rightarrow$ nada se pode afirmar

A confirmação da viabilidade por esta metodologia simples descarta a necessidade da utilização de qualquer método numérico de cálculo do autovalor de PerronFrobenius. Na situação em que $R_{G_{r} \cap G_{c}}^{\max } \geq 1$, não se pode garantir que o sistema seja factível e a certificação sobre a existência do vetor ótimo deve ser feita por outro mecanismo numérico. Neste caso, a diferença entre $R_{G_{r}}^{\max } G_{c}$ e a unidade pode ser entendida como uma medida da incerteza que este método fornece sobre a viabilidade.

A condição suficiente para que os $\mathrm{GC}$ de $\mathbf{H}$ ou $\mathbf{H}^{\mathbf{T}}$ pertençam à região interna do círculo unitário permite verificar a tendência de viabilidade conforme se variam os parâmetros que definem $h_{i j}$.

Em um esquema DS-CDMA com receptor convencional, o desalinhamento temporal entre os usuários que compartilham o canal reverso impede o uso de seqüências de espalhamento ortogonais e, portanto, a SINR na saída de cada filtro casado depende das propriedades de correlação cruzada entre as seqüências utilizadas. De acordo com (1), a potência do usuário interferente $j$ é atenuada por $g_{i j}$. Este ganho é tão menor quanto menor for a correlação cruzada entre os usuários $i$ e $j$ [5, eq. (6)]. Como $h_{i j} \propto g_{i j}$, baixas correlações cruzadas tendem a diminuir os $\mathrm{GC}$ e, portanto, tornar o sistema factivel.

Analogamente, o ganho $g_{i j}$ é influenciado pelo canal de rádio móvel [5, eq. (6)], e, como $h_{i j} \propto g_{i j} g_{i i}{ }^{-1}$, ocorrem flutuações temporais no valor de $R_{G_{r} \cap G_{c}}^{\max }$. A amplitude do sinal transmitido por um determinado usuário sofre desvanecimento de larga escala, $m(t)$, e de pequena escala, $r(t)$ [7]. Desta forma, $g_{i j} \propto\left(m_{i j} r_{i j}\right)^{2}$ e $h_{i j} \propto\left(\frac{m_{i j} r_{i j}}{m_{i i} r_{i i}}\right)^{2}$. Portanto, desvanecimento profundo sobre o sinal de interesse $i$ aliado à interferência construtiva dos multipercursos do usuário $j$ podem fazer com que $R_{G_{r} \cap G_{c}}^{\max }$ atinja valor elevado, indicando tendência à inviabilidade. Adicionalmente, se o usuário $i$ estiver mais próximo da ERB que o $j$, o fator $m_{i j} / m_{i i}$ diminui fazendo com que a incerteza sobre a viabilidade seja menor.

Finalmente, como $h_{i j} \propto \gamma_{i}^{*}$, altos níveis de SINR tendem a aumentar os GC, indicando que o sistema pode tornar-se não factível. Neste caso, relaxações adequadas para atender as distintas qualidades de serviços (QoS - Quality of Service) críticos viabilizam a implementação do controle de potência.

As análises relativas aos DPCA's expostos neste artigo pressupõem que o autovalor de Perron-Frobenius da matriz $\mathbf{H}$ seja menor do que a unidade.

\section{ALGORITMOS DE CONTROLE DE POTÊNCIA DISTRIBUÍDOS}

A solução ótima do problema de alocação satisfaz o seguinte conjunto de equações (ver (2)):

$p_{i}^{*}=\sum_{j \neq i}^{K} h_{i j} p_{j}^{*}+\eta_{i}, i=1, \ldots, K$

Agora, considere uma função ímpar arbitrária $y_{i}=z_{i}(x)$ que tenha valor nulo apenas na origem. Assim, (12) constitui o único ponto de equilíbrio do seguinte sistema dinâmico de tempo contínuo:

$\gtrless_{l}^{\&}=z_{i}\left(-p_{i}+\sum_{j \neq i}^{K} h_{i j} p_{j}+\eta_{i}\right), i=1, \ldots, K$

Na Fig. 1, estrutura-se o diagrama de blocos referente à Eq. (13).

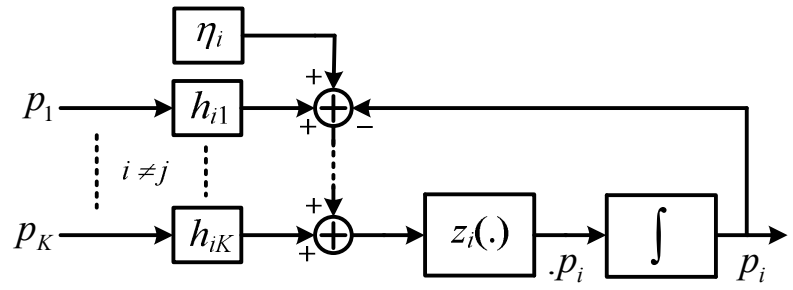

Figura 1. Sistema dinâmico com equilíbrio na solução do problema de alocação.

A reta secante a $p_{i}(t)$ nos instantes $t$ e $\alpha_{i}+t$ é indistingüível da tangente em $t$ quando $\alpha_{i}$ for suficientemente pequeno, supondo que $p_{i}(t)$ seja uma curva suave. Desta forma, vale a aproximação:

$p_{i}\left(t+\alpha_{i}\right) \approx p_{i}(t)+\alpha_{i} z_{i}\left(-p_{i}(t)+\sum_{j \neq i}^{K} h_{i j} p_{j}(t)+\eta_{i}\right)$,

onde $\alpha_{i}>0, i=1, \ldots, K$.

A Eq. (14) consiste no conhecido método de Euler de integração numérica [8].

Do exposto, a seqüência $p_{i}[n]=p_{i}\left(n \alpha_{i}\right), n \in \mathrm{N}$, pode ser obtida recursivamente por:

$p_{i}[n+1]=p_{i}[n]+\alpha_{i} z_{i}\left(-p_{i}[n]+\sum_{j \neq i}^{K} h_{i j} p_{j}[n]+\eta_{i}\right)$

Das definições em (1) e (2), verifica-se que: 


$$
-p_{i}+\sum_{j \neq i}^{K} h_{i j} p_{j}+\eta_{i}=-\left(1-\frac{\gamma_{i}^{*}}{\gamma_{i}}\right) p_{i}
$$

Portanto, (15) equivale a:

$$
p_{i}[n+1]=p_{i}[n]-\alpha_{i} z_{i}\left(\left(1-\frac{\gamma_{i}^{*}}{\gamma_{i}[n]}\right) p_{i}[n]\right)
$$

A recursão em (17) pode ser efetivamente implementada na unidade móvel $i$. Admite-se que o parâmetro $\alpha_{i}$, o nível de QoS quantificado por $\gamma_{i}^{*}$, a função $z_{i}(\cdot)$ e o valor da potência transmitida $p_{i}[n]$ estejam disponíveis na unidade móvel $i\left(\mathrm{M}_{i}\right.$ na Fig. 2). Entretanto, a SINR $\gamma_{i}[n]$ pode ser obtida somente na estação rádio base $\mathrm{B}_{i}$, aquela que demodula o sinal do usuário $i$. Desta forma, para que o usuário $i$ calcule por meio de (17) o próximo nível de potência, $\mathrm{B}_{i}$ precisa estimar $\gamma_{i}[n]$, quantizar o resultado em um número conveniente de bits e, em seguida, realimentá-lo para o usuário $i$ através do canal direto. Todo o processo está esquematizado na Fig. 2.

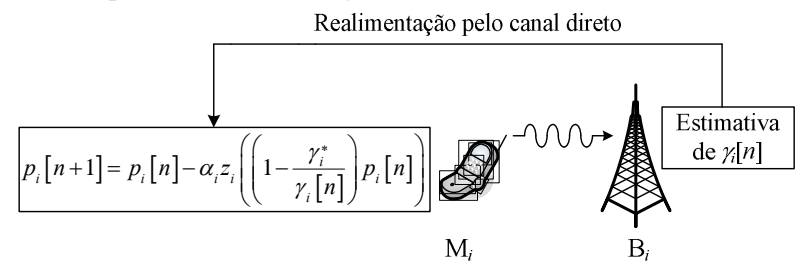

Figura 2. Princípio de funcionamento de um DPCA.

É possível mensurar $\gamma_{i}[n]$ sem o conhecimento efetivo das informações relativas aos usuários interferentes, as quais influenciam em seu valor de acordo com a Eq. (1). Assim, (17) depende apenas de parâmetros locais, permitindo que o controle de potência como um todo funcione de forma distribuida, ou seja, que cada um dos $K$ elos $\mathrm{MB}_{i} \mathrm{~B}_{i}$ realize isoladamente o respectivo controle de potência [5]. Justifica-se, portanto, a denominação algoritmo de controle de potência distribuído (DPCA) para o conjunto de recursões em (17).

Em contraste à estratégia distribuída, o método centralizado para resolver o problema de alocação ótima consiste em reunir todos os elementos da matriz $\mathbf{H}$ e do vetor $\eta$ em alguma entidade que execute a operação em (3) e posteriormente "entregue" os valores ótimos das potências. Desta forma, o esquema centralizado necessita da estimação de cada elemento de $\mathbf{H}$ e de $\eta$, do tráfego dos valores destes elementos e, finalmente, de uma inversão matricial. Portanto, a complexidade de implementação da metodologia centralizada é acentuadamente maior do que a distribuída. Quando o número de usuários ativos no sistema multicelular for elevado, a quantidade de operações necessárias para a inversão matricial, o volume de estimações e o tráfego na rede desses valores estimados atingem proporções que tornam impraticável a estratégia centralizada.

\subsection{DPCAS COMPARADOS}

O DPCA desenvolvido por Foschini e Miljanic [1], aqui denominado DPCA clássico, é obtido simplesmente substituindo: $z_{i}(x)=x, \quad \alpha_{i}=\alpha, \quad i=1, \ldots, K$,

em (17); portanto, o processo iterativo para o DPCA clássico resulta:

$p_{i}[n+1]=p_{i}[n]-\alpha\left(1-\frac{\gamma_{i}^{*}}{\gamma_{i}[n]}\right) p_{i}[n], i=1, \ldots, K$

No trabalho publicado por Uykan e Koivo [4] sugere-se que o controle de potência equivale a um problema de projeto de controlador, conforme o modelo contínuo da Fig. 1. Para sua síntese, simplesmente substitui-se:

$z_{i}(x)=\tanh \left(\frac{x}{2}\right), \quad \alpha_{i}=\alpha, i=1, \ldots, K$,

em (17). Assim, o DPCA sigmoidal é dado por [4]:

$p_{i}[n+1]=p_{i}[n]-\alpha \tanh \left(\frac{1}{2}\left(1-\frac{\gamma_{i}^{*}}{\gamma_{i}[n]}\right) p_{i}[n]\right), i=1, \ldots, K$

Observe que o desenvolvimento feito em [4] inclui ainda uma constante multiplicativa ajustável no argumento da função $\tanh ($.$) .$

A utilização de função sigmoidal resulta em convergência quadrática na vizinhança do ponto ótimo [4] e desde que $\rho_{\mathbf{H}}<1$, o algoritmo sigmoidal converge para qualquer vetor de potência inicial.

\section{DPCA PROPOSTO}

O modelo dinâmico atribuído a P.F.Verhulst é dado por [2]

$\&=z(p)=p\left(1-\frac{p}{p^{*}}\right)$

Verhulst formulou esta equação na tentativa de descrever a evolução temporal do número de indivíduos, denotado por $p(t)$, de uma determinada espécie biológica. Seu modelo supõe que o alimento disponível e o espaço físico ocupado sejam finitos. Essa limitação de recursos impede que a população cresça de maneira ilimitada. Tais considerações são levadas em conta no termo que provoca diminuição da taxa de crescimento conforme a população aumenta. Integrando-se (22) analiticamente obtém-se:

$p(t)=\frac{\exp (t) p(0) p^{*}}{p^{*}+p(0)[\exp (t)-1]}$

O comportamento assintótico desta solução é dado por:

$\lim _{t \rightarrow+\infty} p(t)=p^{*}$

para qualquer condição inicial $p(0)$ estritamente positiva, conforme evidenciado na Fig. 3.

Quando $p(0)$ for maior que $p^{*}$, o sinal de $d p / d t$ será negativo, e $p(t)$ decrescerá de forma que $p(+\infty)=p^{*}$. Caso contrário, $d p / d t$ será positivo, e $p(t)$ aumentará monotonicamente em direção ao seu valor assintótico [2]. 


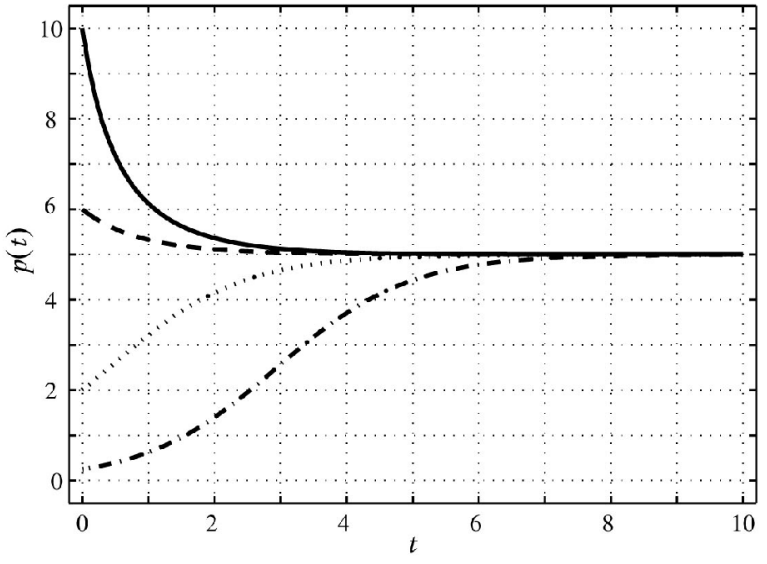

Figura 3. Curvas $p(t)$ parametrizadas por diferentes condições iniciais.

Supondo que existam várias espécies sob análise, é conveniente que se reescreva (22) em um formato matricial:

$\mathbf{p}<=\mathbf{z}(\mathbf{p})=\mathbf{p} \otimes\left(\mathbf{u}-\mathbf{p} \Theta \mathbf{p}^{*}\right)$

sendo que $\mathbf{p}=\left[\begin{array}{ll}p_{1} \mathrm{~L} & p_{K}\end{array}\right]^{T}, \mathbf{u}=\left[\begin{array}{ll}1 \mathrm{~L} & 1\end{array}\right]^{T}$ e $\mathbf{p}^{*}=\left[\begin{array}{lll}p_{1}^{*} \mathrm{~L} & p_{K}^{*}\end{array}\right]^{T}$. Os operadores empregados em (25) representam as operações elementares $[\mathbf{A} \otimes \mathbf{B}]_{i j}=[\mathbf{A}]_{i j}[\mathbf{B}]_{i j}$ e $[\mathbf{A} \Theta \mathbf{B}]_{i j}=[\mathbf{A}]_{i j} /[\mathbf{B}]_{i j}$, sendo

$\mathbf{A}$ e $\mathbf{B}$ matrizes com dimensões iguais.

Impondo que a evolução do vetor de potências possa ser regida pelo modelo biológico de (25) e admitindo-se que p esteja suficientemente próximo de $\mathbf{p} *$ pode-se escrever, a partir de (2):

$\mathbf{p}^{*} \approx \mathbf{H p}+\boldsymbol{\eta}$

Substituindo (26) no modelo de Verhulst, adaptado à forma matricial em (25), obtém-se:

$\boldsymbol{\beta}=\mathbf{p} \otimes[\mathbf{u}-\mathbf{p} \Theta(\mathbf{H p}+\boldsymbol{\eta})]$

Portanto:

$\mathbf{z}(-(\mathbf{I}-\mathbf{H}) \mathbf{p}+\boldsymbol{\eta})=\mathbf{p} \otimes[\mathbf{u}-\mathbf{p} \Theta(\mathbf{H p}+\boldsymbol{\eta})]$

Neste caso, o formato do mapeamento $\mathbf{y}=\mathbf{z}(\mathbf{x})$ depende da matriz $\mathbf{H}$ e do vetor $\eta$. Considerando o ponto $K$ dimensional $\mathbf{x}_{0}=-\left(\mathbf{I}-\mathbf{H}_{0}\right) \mathbf{p}_{0}+\boldsymbol{\eta}_{0}$, obtém-se, após a aplicação de $\mathbf{z}\left(\right.$.), o ponto $\mathbf{y}_{0}=\mathbf{p}_{0} \otimes\left[\mathbf{u}-\mathbf{p}_{0} \Theta\left(\mathbf{H}_{0} \mathbf{p}_{0}+\boldsymbol{\eta}_{0}\right)\right]$. Alterando $\mathbf{H}_{0}$ para $\mathbf{H}_{1}$ e $\eta_{0}$ para $\eta_{1}$ é sempre possível encontrar um vetor $\mathbf{p}_{1}$ de forma que $\mathbf{x}_{0}=-\left(\mathbf{I}-\mathbf{H}_{0}\right) \mathbf{p}_{0}+\boldsymbol{\eta}_{0}=-\left(\mathbf{I}-\mathbf{H}_{1}\right) \mathbf{p}_{1}+\boldsymbol{\eta}_{1}=\mathbf{x}_{1}$. Assim, $\quad \mathbf{y}_{1}=\mathbf{z}\left(\mathbf{x}_{1}\right)=\mathbf{p}_{1} \otimes\left[\mathbf{u}-\mathbf{p}_{1} \Theta\left(\mathbf{H}_{1} \mathbf{p}_{1}+\boldsymbol{\eta}_{1}\right)\right] \neq \mathbf{y}_{0}$. Portanto, para o DPCA proposto, a modificação da matriz $\mathbf{H}$ e/ou do vetor $\eta$ altera o mapeamento $\mathbf{y}=\mathbf{z}(\mathbf{x})$. No DPCA clássico e no sigmoidal, o mapeamento $\mathbf{y}=\mathbf{z}(\mathbf{x})$ é imutável.

Analogamente aos DPCA's clássico e sigmoidal, o comportamento do DPCA proposto pode ser sintetizado a partir de um sistema de controle. Entretanto, para este novo DPCA, o diagrama de controle apresentado na Fig. 1 é insuficiente e deve ser modificado de forma a incorporar a variabilidade do mapeamento $\mathbf{y}=\mathbf{z}(\mathbf{x})$. Esta modificação está representada na Fig. 4 pela presença do bloco e seta tracejados, os quais indicam que $z_{i}($.$) sofre influência de$ cada $h_{i j, i \neq j}$ e de $\eta_{i}$.

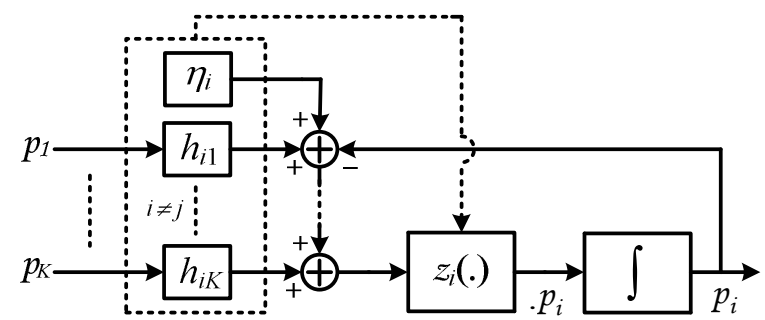

Figura 4. Comportamento sistêmico do DPCA proposto.

Discretizando (27) pelo método NIE, obtém-se:

$\mathbf{p}[n+1]=\mathbf{F}(\mathbf{p}[n])=\mathbf{p}[n]+\alpha \mathbf{p}[n] \otimes\{\mathbf{u}-\mathbf{p}[n] \Theta[\mathbf{H p}[n]+\boldsymbol{\eta}]\}$

Portanto, calcula-se o elemento $i$ do vetor $\boldsymbol{F}(p)$ por:

$F_{i}(\mathbf{p})=(1+\alpha) p_{i}-\frac{\alpha}{\gamma_{i}^{*}} \mu_{i}(\mathbf{p}) p_{i}^{2}, \mu_{i}(\mathbf{p})=\frac{g_{i i}}{\sum_{j \neq i} g_{i j} p_{j}+\sigma_{i}^{2}}$

A equação matricial (29) é equivalente ao conjunto de equações escalares:

$p_{i}[n+1]=(1+\alpha) p_{i}[n]-\alpha\left[\frac{\gamma_{i}[n]}{\gamma_{i}^{*}}\right] p_{i}[n], i=1, \mathrm{~L}, K$

As equações em (31) consistem na versão implementável do DPCA proposto. Salienta-se que o diagrama de controle da Fig. 4 é apenas uma forma sistemática de visualizar como ocorre a evolução da potência transmitida, não correspondendo à estrutura que deve ser efetivamente implementada na prática. Observa-se que a implementação direta do esquema da Fig. 4 exigiria o conhecimento exato de cada $h_{i j, i \neq j}, \eta_{i}$ e $z_{i}($.) e, conseqüentemente, seria um procedimento centralizado. De fato, se $h_{i j, i \neq j}, \eta_{i}$ e $z_{i}($. fossem conhecidos exatamente e se a estimação da SINR $\gamma_{i}(n)$ fosse perfeita em cada iteração $n$ de (31), o sistema de controle da Fig. 4 produziria o mesmo resultado que (31).

Observa-se em (31) que a atualização da potência transmitida de cada usuário depende apenas de parâmetros referentes ao mesmo, ou seja, da sua própria SINR estimada, do seu nível de potência transmitido na iteração anterior e de sua meta de QoS. Esse aparente desacoplamento entre os usuários decorre, evidentemente, do fato de se estimar a SINR diretamente e não efetuar seu cálculo pela relação que a define, equação (1). A obtenção da SINR aplicando-se (1) exigiria processos dispendiosos de estimação para os ganhos de canal dos interferentes e para o ganho do usuário de interesse, tornando a implementação do algoritmo mais complexa.

As considerações mencionadas no parágrafo anterior são típicas de algoritmos distribuídos. Entretanto, o DPCA proposto, definido pelas iterações em (31), apresenta uma característica singular: no termo entre colchetes, a SINR estimada está no numerador da divisão. Esta propriedade torna a sensibilidade do algoritmo face a erros de estimação independente do nível verdadeiro da SINR. Sabe-se que função do tipo $\varphi=\kappa \gamma^{-1}$ (SINR no denominador é inerente aos algoritmos distribuídos propostos na literatura atual, como o clássico [1] e o sigmoidal [4]) possui incremento diferencial $d \varphi=-\kappa \gamma^{-2} \mathrm{~d} \gamma$, ou seja, o montante da variação de $\varphi$ para pequenos desvios de $\gamma$ depende do valor real de $\gamma$; enquanto que para a função do tipo $\varphi=\kappa \gamma($ SINR no 
numerador, caso do DPCA proposto), tem-se $\mathrm{d} \varphi=\kappa \mathrm{d} \gamma$, não havendo influência, portanto, do nível verdadeiro de $\gamma$ sobre a variação de $\varphi$. No algoritmo sigmoidal [4], observa-se que o resultado da operação na qual $\gamma$ está no denominador é ainda argumento de uma função do tipo tangente hiperbólica. Evidentemente, esta função hiperbólica influencia, mas não anula, a relação entre o valor real de $\gamma$ e o comportamento do algoritmo na presença de medidas imperfeitas de SINR.

\section{CONVERGÊNCIA PARA O DPCA PROPOSTO}

A região do hiperespaço cujos pontos sejam levados ao ponto ótimo $p^{*}$ por sucessivas aplicações de $\boldsymbol{F}($.) denominase domínio atrator ou bacia de atração do mapeamento $p$ $(n+1)=\boldsymbol{F}(p(n))$. Evidentemente, determinar a localização desta região, ou parte dela, evita que se faça uma escolha inadequada de condição inicial $p(0)$ em (29). Utilizando-se de 11 proposições, descreve-se a seguir a consistência de um subconjunto da bacia de atração para o algoritmo DPCA proposto; as proposições são demonstradas e confirmadas por meio de gráficos.

\subsection{SUBCONJUNTO ATRATOR PARA O DPCA PROPOSTO}

O subconjunto do domínio atrator para o DPCA proposto resulta da concatenação das proposições subseqüentes.

Proposição 1: A solução ótima $p^{*}$ do problema de alocação é ponto fixo de $\boldsymbol{F}(p)$.

Prova: Para o ponto ótimo $p^{*}$ tem-se $p_{i}^{*}=\left(\mu_{i}\left(p^{*}\right)\right)^{-1} \gamma_{i}^{*}$. Portanto, das equações apresentadas em (30), obtém-se: $F_{i}\left(p^{*}\right)=(1+\alpha) p_{i}^{*}-\alpha \mu_{i}\left(p^{*}\right)\left(\gamma_{i}^{*}\right)^{-1}\left(p_{i}^{*}\right)^{2}=p_{i}^{*}$

Proposição 2: Considere o poliedro $P_{\alpha}=\{p:(\beta \mathbf{I}-\mathbf{H}) p \leq \eta$, $p>0\}, \beta=(2 \alpha)(1+\alpha)^{-1}$. Se $p^{\prime} \geq p^{\prime \prime}$ e ambos estiverem em $P_{\alpha}$, então $\boldsymbol{F}\left(p^{\prime}\right) \geq \boldsymbol{F}\left(p^{\prime \prime}\right)$.

Prova: Evidentemente, é suficiente demonstrar que $\boldsymbol{F}(p)$ cresce monotonicamente em $P_{\alpha}$. Para $i \neq j$ tem-se $\partial \mu_{i}(p) / \partial p_{j}<0$ e, portanto, o elemento $i$ do vetor $\boldsymbol{F}(p)$, dado por $F_{i}(p)=(1+\alpha) p_{i}-\alpha \mu_{i}(p)\left(\gamma_{i}^{*}\right)^{-1} p_{i}^{2}$, aumenta de forma estrita quando cresce qualquer potência interferente $p_{j}$. Na direção $p_{i}$, entretanto, a função de várias variáveis $F_{i}(p)$ consiste numa parábola côncava que passa pela origem e atinge valor máximo no ponto $p_{i}=(1+\alpha)\left(2 \alpha \mu_{i}(p)\right)^{-1} \gamma_{i}{ }^{*}$. Portanto, $F_{i}(p)$ cresce no domínio definido pela desigualdade $p_{i} \leq(1+\alpha)\left(2 \alpha \mu_{i}(p)\right)^{-1} \gamma_{i}{ }^{*}$. A definição de $\mu_{i}(p)$ na eq. (30) implica que $p_{i}=(1+\alpha)\left(2 \alpha \mu_{i}(p)\right)^{-1} \gamma_{i}{ }^{*}$ representa a equação de um hiperplano. Assim, $\boldsymbol{F}(p)$ cresce monotonicamente no poliedro $0<p_{i} \leq(1+\alpha)\left(2 \alpha \mu_{i}(p)\right)^{-1} \gamma_{i}{ }^{*}$, $i=1,2, \ldots, K$, o qual equivale à inequação matricial $(\beta \mathbf{I}-\mathbf{H}) p \leq \eta, p>0$, onde $\beta=(2 \alpha)(1+\alpha)^{-1}$.

Proposição 3: $p^{*} \in P_{\alpha}$ para $0<\alpha \leq 1$.

Prova: O poliedro $P_{\alpha}$ contém a solução do problema de alocação $p^{*}=(\mathrm{I}-\mathrm{H})^{-1} \eta$ se $(\beta \mathbf{I}-\mathbf{H}) p^{*} \leq \eta$. Assim, basta verificar se $\beta p^{*} \leq \eta+\mathbf{H} p^{*}=p^{*}$. Como $\beta=(2 \alpha)(1+\alpha)^{-1}$, tem-se $0<\beta \leq 1$ para $0<\alpha \leq 1$ e, portanto, $\beta p^{*} \leq p^{*}$.

Proposição 4: Se $p^{\prime} \in P_{\alpha}$ e $0<\lambda<1$, então $\lambda p^{\prime} \in P_{\alpha}$.

Prova: Se $p^{\prime} \in P_{\alpha}$, então $(\beta \mathbf{I}-\mathbf{H}) p^{\prime} \leq \eta$ e $p^{\prime}>0$. Logo, $(\beta \mathbf{I}-\mathbf{H}) \lambda p^{\prime} \leq \lambda \eta<\eta$ e $\lambda p^{\prime}>0$.

Proposição 5: Para todo $0<\lambda<1, \boldsymbol{F}(\lambda p)>\lambda \boldsymbol{F}(p)$. E, para todo $\lambda \geq 1, \lambda \boldsymbol{F}(p) \geq \boldsymbol{F}(\lambda p)$.

Prova: Das equações apresentadas em (30) fica evidente que $F_{i}(\lambda p)-\lambda F_{i}(p)=\left[\mu_{i}(p)-\lambda \mu_{i}(\lambda p)\right] \lambda \alpha\left(\gamma_{i}^{*}\right)^{-1} p_{i}^{2}$. Lembrando que $\mu_{i}(p)=g_{i i}\left(\mathrm{~S}_{i}+\sigma_{i}^{2}\right)^{-1}$, onde $\mathrm{S}_{i}=\Sigma_{j \neq i} g_{i j} p_{j}$, obtém-se $\lambda \mu_{i}(\lambda p) / \mu_{i}(p)=\left(\mathrm{S}_{i}+\sigma_{i}^{2}\right) /\left(\mathrm{S}_{i}+\lambda^{-1} \sigma_{i}^{2}\right)$. Assim, para $0<\lambda<1$, $\mu_{i}(p)-\lambda \mu_{i}(\lambda p)>0$ e, para $\lambda \geq 1, \mu_{i}(p)-\lambda \mu_{i}(\lambda p) \leq 0$.

Proposição 6: $\mathrm{O}$ poliedro $P_{\alpha}$ não contém pontos fixos distintos.

Prova: Por hipótese, admite-se que os vetores estritamente positivos $p^{\prime}$ e $p^{\prime \prime}$ sejam pontos fixos distintos contidos em $P_{\alpha}$. Sem perda de generalidade, pode-se assumir que existe pelo menos um $i$ tal que $p_{i}^{\prime \prime}<p_{i}^{\prime}$. Então, existe $0<\lambda<1$ tal que $p^{\prime \prime} \geq \lambda p^{\prime}$, com igualdade $p_{i}^{\prime \prime}=\lambda p_{i}^{\prime}$ para algum $i$. Das Proposições 2 , 4 e 5 , obtém-se:

$p_{i}^{\prime \prime}=F_{i}\left(p^{\prime \prime}\right) \geq F_{i}\left(\lambda p^{\prime}\right)>\lambda F_{i}\left(p^{\prime}\right)=\lambda p_{i}^{\prime}$

Como $p_{i}^{\prime \prime}=\lambda p_{i}^{\prime}$, tem-se uma contradição e, portanto, a hipótese da existência de pontos fixos distintos em $P_{\alpha}$ é falsa.

Proposição 7: Considere $0<\alpha \leq 1$. Neste caso, se $p \in P_{\alpha}$, então $\boldsymbol{F}^{n}(p) \in P_{\alpha}$.

Prova: Usa-se $\boldsymbol{F}^{n}(p)$ para representar $\boldsymbol{F}(\boldsymbol{F}(\boldsymbol{F}(\boldsymbol{F}(p))))$, onde $n$ corresponde ao número de vezes que se aplica a função $\boldsymbol{F}($.$) . Portanto, a iteração p(m+1)=\boldsymbol{F}(p(m))$ pode ser escrita como $p(n)=\boldsymbol{F}^{n}(p)$, onde $p=p(0)$.

Dado que $p \in P_{\alpha}$, para demonstrar que a seqüência $\boldsymbol{F}^{n}(p)$ fica confinada no poliedro $P_{\alpha}$, é suficiente mostrar que $\boldsymbol{F}(p) \in P_{\alpha}$, pois o vetor $\boldsymbol{F}(p)$ pode ser interpretado como um novo $p$.

A definição $\boldsymbol{d}(p)=\boldsymbol{F}(p)-p$ aliada à eq. (30) resulta em:

$d_{i}(p)=F_{i}(p)-p_{i}=\alpha p_{i}\left(1-\mu_{i}(p) p_{i} / \gamma_{i}^{*}\right)$

De acordo com esta equação, a variação da potência transmitida pelo usuário $i$ será positiva se a meta $\gamma_{i}{ }^{*}$ for maior que a $\operatorname{SINR}_{i}=\mu_{i}(p) p_{i}$ e negativa caso contrário. Notese que este fato está coerente com o objetivo do algoritmo, que consiste em encontrar o vetor de potências que torna a SINR de cada usuário igual à respectiva meta de QoS.

Para que um determinado vetor $p$ não seja exterior ao poliedro $P_{\alpha}$, qualquer $p_{i}$ deve ser no máximo igual a $(1+\alpha)\left(2 \alpha \mu_{i}(p)\right)^{-1} \gamma_{i}{ }^{*}$. Da Eq. (33), pode-se afirmar que, na região entre os hiperplanos $p_{i}=(1+\alpha)\left(2 \alpha \mu_{i}(p)\right)^{-1} \gamma_{i}{ }^{*}$, com $0<\alpha \leq 1$, e $p_{i}=\left(\mu_{i}(p)\right)^{-1} \gamma_{i}^{*}$, a variação $d_{i}(p)$ possui sinal negativo. Logo, qualquer ponto em tal região se afasta da fronteira $p_{i}=(1+\alpha)\left(2 \alpha \mu_{i}(p)\right)^{-1} \gamma_{i}{ }^{*}$, tendendo ao interior do poliedro $P_{\alpha}$. Evidentemente, estas observações são válidas para qualquer índice $i$, ou seja, precede cada fronteira $i$ do poliedro $P_{\alpha}$ uma região com $d_{i}(p)<0$. Assim, $P_{\alpha}$ possui uma camada $\boldsymbol{C}_{\alpha}$ que reveste internamente toda sua extensão 
limítrofe de tal sorte que todo $p$ nela localizado tende ao interior do poliedro ao se aplicar $\boldsymbol{F}\left(\right.$.), ou seja, $p \in \boldsymbol{C}_{\alpha} \Rightarrow$ $\boldsymbol{F}(p) \in P_{\alpha}$.

Para garantir o confinamento em $P_{\alpha}$, deve-se mostrar ainda que não existe possibilidade de algum $p$ "saltar" diretamente tal camada ao se aplicar $\boldsymbol{F}\left(\right.$.). Para tanto, considere $m_{\alpha}$ o conjunto que resta quando se retira do poliedro $P_{\alpha}$ essa sua camada externa $\boldsymbol{C}_{\alpha}$, ou seja, $P_{\alpha}=\boldsymbol{C}_{\alpha} \cup m_{\alpha}$ e $\boldsymbol{C}_{\alpha} \cap m_{\alpha}=\varnothing$. Se todo $p$ em $M_{\alpha}$ resultar em $\boldsymbol{F}(p)$ no interior de $P_{\alpha}$, então o "salto" mencionado não ocorre.

Quando todo $F_{i}(p)$ for inferior à respectiva fronteira $(1+\alpha)\left[2 \alpha \mu_{i}(\boldsymbol{F}(p))\right]^{-1} \gamma_{i}{ }^{*}$, o vetor $\boldsymbol{F}(p)$ localiza-se em $P_{\alpha}$. Assim, é necessário que:

$r_{i}=(1+\alpha)\left[2 \alpha \mu_{i}(\boldsymbol{F}(p))\right]^{-1} \gamma_{i}{ }^{*}-F_{i}(p) \geq 0$, para $i=1, \ldots, K$

Portanto, observando as definições em (30), deve-se ter, para todo $i$ :

$r_{i}=\alpha\left(\xi_{i}(p)\right)^{-1} p_{i}^{2}-(1+\alpha) p_{i}+(1+\alpha)(2 \alpha)^{-1} \xi_{i}(\boldsymbol{F}(p)) \geq 0$,

$\operatorname{com} \xi_{i}(p)=\gamma_{i}^{*} / \mu_{i}(p)$

$\mathrm{O}$ discriminante $\zeta(\alpha)$ desta parábola vale:

$\zeta(\alpha)=(1+\alpha)\left[(1+\alpha)-2 \xi_{i}(\boldsymbol{F}(p))\left(\xi_{i}(p)\right)^{-1}\right]$

$\mathrm{O}$ vetor $\boldsymbol{d}(p)=\boldsymbol{F}(p)-p$ não possui elemento negativo para todo ponto $p$ em $m_{\alpha}$. Assim, $\boldsymbol{F}(p) \geq p$ e, conseqüentemente, $\mu_{i}(p)\left[\mu_{i}(\boldsymbol{F}(p))\right]^{-1} \geq 1$. Mostra-se que o lado esquerdo desta desigualdade equivale a $\xi_{i}(\boldsymbol{F}(p))\left[\xi_{i}(p)\right]^{-1}$. Logo, o discriminante dado na eq. (36) não atinge valor positivo para $0<\alpha \leq 1$. Portanto, a condição $r_{i} \geq 0$ na Eq. (35) sempre é satisfeita para qualquer $i$, o que equivale a afirmar que o vetor $\boldsymbol{F}(p)$ não ultrapassa nenhuma fronteira de $P_{\alpha}$ quando $p$ estiver em $M_{\alpha}$, ou seja, pode-se escrever que $p$ $\in M_{\alpha} \Rightarrow \boldsymbol{F}(p) \in P_{\alpha}$.

Proposição 8: Dado que $0<\alpha \leq 1$ e $0<\lambda<1$, a seqüência $p(n)=\boldsymbol{F}^{n}\left(\lambda p^{*}\right)$ converge para o ponto ótimo $p^{*}$ de forma monotônica.

Prova: As Proposições 1 e 3 garantem que $p^{*}$ é um ponto fixo localizado em $P_{\alpha}$. A desigualdade $p^{*}>\lambda p^{*}$, aliada às Proposições 2, 4 e 5, resulta em:

$p^{*}=\boldsymbol{F}\left(p^{*}\right)>\boldsymbol{F}\left(\lambda p^{*}\right)>\lambda \boldsymbol{F}\left(p^{*}\right)=\lambda p^{*}$

$\mathrm{O}$ confinamento (Proposição 7 ) na região de monotonicidade $P_{\alpha}$ assegura que aplicações sucessivas de $\boldsymbol{F}($.) em cada termo de (37) não alteram a ordem das desigualdades, ou seja:

$p^{*}>\boldsymbol{F}^{n+1}\left(\lambda p^{*}\right)>\boldsymbol{F}^{n}\left(\lambda p^{*}\right)$

De forma equivalente:

$p^{*}>p(n+1)>p(n), \operatorname{com} p(0)=\lambda p^{*}$

Portanto, a seqüência $p(n)=\boldsymbol{F}^{n}\left(\lambda p^{*}\right)$ é estritamente crescente e limitada acima por $p^{*}$. Como há somente este ponto fixo em $P_{\alpha}$, afirma-se que:

$\lim _{n \rightarrow \infty} \boldsymbol{F}^{n}\left(\lambda p^{*}\right)=p^{*}$

A unicidade de $p^{*}$ em $P_{\alpha}$ decorre da Proposição 6 .

Proposição 9: Considerando $0<\alpha \leq 1, \lambda p^{*} \in P_{\alpha}$ para todo $\lambda \geq 1$ que satisfaz $\mathcal{T}(\lambda)=\max _{i=1, \ldots, K}\left(\beta \lambda \mu_{i}\left(\lambda p^{*}\right) / \mu_{i}\left(p^{*}\right)\right) \leq 1$.

Prova: Da Eq. (30):

$\partial F_{i}(p) /\left.\partial p_{i}\right|_{p=\lambda p^{*}}=(1+\alpha)-2 \alpha \lambda \mu_{i}\left(\lambda p^{*}\right) / \mu_{i}\left(p^{*}\right)$
Se $\partial F_{i}(p) /\left.\partial p_{i}\right|_{p=\lambda p^{*}}<0$ para pelo menos um $i$, então $\lambda p^{*} \notin P_{\alpha}$. Deste fato aliado à Eq. (41), infere-se que $\lambda$ deve satisfazer todas as seguintes desigualdades para que o ponto $\lambda p^{*}$ localize-se em $P_{\alpha}$ :

$\beta \lambda \mu_{i}\left(\lambda p^{*}\right) / \mu_{i}\left(p^{*}\right) \leq 1, i=1, \ldots, K$, onde $\beta=(2 \alpha)(1+\alpha)^{-1}$ (42)

Portanto, multiplicar $p^{*}$ por $\lambda \geq 1$ não resulta em ponto exterior ao poliedro $P_{\alpha}, 0<\alpha \leq 1$, se $\lambda$ for tal que:

$\mathcal{T}(\lambda)=\max _{i=1, \ldots, K}\left(\beta \lambda \mu_{i}\left(\lambda p^{*}\right) / \mu_{i}\left(p^{*}\right)\right) \leq 1$

Proposição 10: Considere $0<\alpha \leq 1$ e um determinado $\lambda \geq 1$ satisfazendo o critério $\tau(\lambda) \leq 1$. Nestas condições, a seqüência $p(n)=\boldsymbol{F}^{n}\left(\lambda p^{*}\right)$ converge monotonicamente para o ponto ótimo $p^{*}$

Prova: Das Proposições 1 e 3 segue que $\boldsymbol{F}\left(p^{*}\right)=p^{*} \in P_{\alpha}$. Associando $\lambda p^{*} \geq p^{*}$ às Proposições 2,5 e 9, obtém-se:

$p^{*}=\boldsymbol{F}\left(p^{*}\right) \leq \boldsymbol{F}\left(\lambda p^{*}\right) \leq \lambda \boldsymbol{F}\left(p^{*}\right)=\lambda p^{*}$

Pela Proposição 7 , ocorre confinamento na região de monotonicidade $P_{\alpha}$. Portanto, sucessivas aplicações de $\boldsymbol{F}($.) em cada termo de (44) não alteram a ordem das desigualdades. Assim:

$p^{*} \leq \boldsymbol{F}^{n+1}\left(\lambda p^{*}\right) \leq \boldsymbol{F}^{n}\left(\lambda p^{*}\right)$

De forma equivalente:

$p^{*} \leq p(n+1) \leq p(n), p(0)=\lambda p^{*}, \lambda \geq 1$ e $\mathcal{T}(\lambda) \leq 1$

Portanto, a seqüência $p(n)=\boldsymbol{F}^{n}\left(\lambda p^{*}\right)$ é decrescente e limitada inferiormente por $p^{*}$. Pela Proposição 6 , este ponto fixo é único em $P_{\alpha}$. Portanto:

$\lim _{n \rightarrow \infty} \boldsymbol{F}^{n}\left(\lambda p^{*}\right)=p^{*}$

Proposição 11: Para $0<\alpha \leq 1$, a seqüência $p(n)=\boldsymbol{F}^{n}(p)$ converge para a solução ótima $p^{*}$ para todo $p \in P_{\alpha}$ não maior do que $\lambda_{2} p^{*}$, onde $\lambda_{2}$ é o maior $\lambda \geq 1$ que satisfaz o critério $\mathcal{T}(\lambda) \leq 1$.

Prova: Para qualquer $p$ inicial positivo contido em $P_{\alpha}$ pode ser encontrado um $\lambda_{1}$ positivo e suficientemente menor do que a unidade de forma que $\lambda_{1} p^{*} \leq p$. Portanto, pode-se estabelecer a ordenação:

$\lambda_{1} p^{*} \leq p \leq \lambda_{2} p^{*}$

As Proposições 4 e 9 garantem que estes limites pertencem a $P_{\alpha}$. O confinamento (Proposição 7 ) na região de monotonicidade $P_{\alpha}$ assegura que sucessivas aplicações de $\boldsymbol{F}$ (.) nos termos em (48) não alteram a ordem das desigualdades. Logo:

$\boldsymbol{F}^{n}\left(\lambda_{1} p^{*}\right) \leq \boldsymbol{F}^{n}(p) \leq \boldsymbol{F}^{n}\left(\lambda_{2} p^{*}\right)(49)$

Pela Proposição $8, \lim _{n \rightarrow \infty} \boldsymbol{F}^{n}\left(\lambda_{1} p^{*}\right)=p^{*}$ e, pela Proposição $10, \lim _{n \rightarrow \infty} \boldsymbol{F}^{n}\left(\lambda_{2} p^{*}\right)=p^{*}$. Então:

$\lim _{n \rightarrow \infty} \boldsymbol{F}^{n}(p)=p^{*}$

Assim, mostrou-se que o conjunto $B=P_{\alpha} \cap\left\{p: p \leq \lambda_{2} p^{*}\right\}$, onde $0<\alpha \leq 1$ e $\lambda_{2}$ o máximo $\lambda \geq 1$ que satisfaz $\mathcal{T}(\lambda) \leq 1$, consiste em um subconjunto da bacia de atração do algoritmo recursivo definido em (29).

Serviu de modelo para a construção de toda essa estrutura lógica, a metodologia de análise da convergência de algoritmos de ponto fixo elaborada em [10]. 


\subsection{ANÁLISE GRÁFICA DA CONVERGÊNCIA}

A percepção de que o ponto fixo do algoritmo proposto localiza-se, quando se tem $0<\alpha \leq 1$, na região crescente de $\boldsymbol{F}(p)$ decorre, primordialmente, das propriedades gráficas apresentadas na Fig. 5. A análise do comportamento de $F_{i}(p)=(1+\alpha) p_{i}-\alpha \mu_{i}(p)\left(\gamma_{i}^{*}\right)^{-1}\left(p_{i}\right)^{2}$ na direção $p_{i}$ pode ser realizada convertendo tal função escalar de várias variáveis em um conjunto de curvas no plano cartesiano. Para tanto, basta interpretar a função multivariável $\mu_{i}(p)$ como sendo um simples parâmetro $\mu_{i}$ e, então, associar a cada valor deste uma curva $f_{i}\left(p_{i}\right)=(1+\alpha) p_{i}-\alpha \mu_{i}\left(\gamma_{i}^{*}\right)^{-1}\left(p_{i}\right)^{2}$, conforme indicado na Fig. 5.

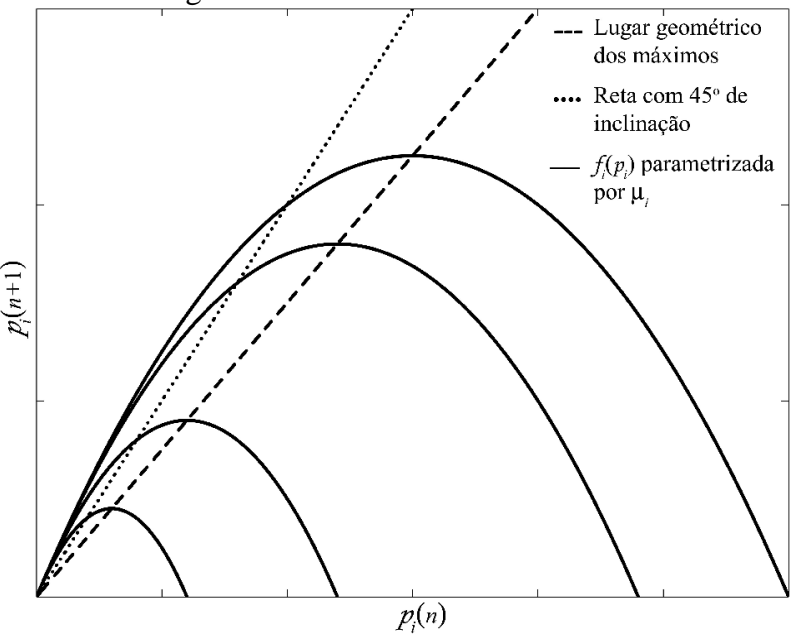

Figura 5. Projeção da função $F_{i}(p)$ no plano cartesiano.

O lugar geométrico dos máximos destas parábolas côncavas do usuário $i$ localiza-se sobre a reta $0,5(1+\alpha) p_{i}$. Logo, para $0<\alpha \leq 1$, todos os máximos estão abaixo da reta que possui $45^{\circ}$ de inclinação, independentemente do valor assumido por $\mu_{i}$. Portanto, o ponto fixo de cada parábola $f_{i}\left(p_{i}\right)$ do usuário $i$ está necessariamente na respectiva região crescente na Fig. 5.

A potência ótima $p_{i}^{*}$ consiste no ponto fixo da parábola que tem parâmetro $\mu_{i}=\mu_{i}\left(p^{*}\right)$. Do parágrafo anterior, infere-se que tal ponto fixo está na região crescente da correspondente curva $f_{i}\left(p_{i}\right)$ quando $0<\alpha \leq 1$. Portanto:

$\partial F_{i}(p) /\left.\partial p_{i}\right|_{p=p^{*}} \geq 0$, para $0<\alpha \leq 1$

Evidentemente, esta argumentação estende-se para todos os usuários, ou seja, vale para $i=1, \ldots, K$. Adicionalmente, para qualquer $p$, verifica-se de imediato que $\partial F_{i}(p) / \partial p_{j}>0$, sendo $i \neq j$. Este fato, aliado às observações acima, permite declarar que:

$\partial F_{i}(p) /\left.\partial p_{j}\right|_{p=p^{*}} \geq 0$,

para quaisquer índices $i$ e $j$ positivos não maiores que $K$.

Esta constatação garante que o ponto fixo $p^{*}$ localiza-se no domínio de crescimento da função vetorial $\boldsymbol{F}(p)$ quando $0<\alpha \leq 1$, fato este que motivou a estruturação do poliedro $P_{\alpha}$ realizada durante a demonstração da Proposição 2.

A seguir, para a explanação gráfica das demais proposições, idealiza-se um cenário com apenas dois usuários compartilhando o canal reverso. Este carregamento com mínima quantidade de interferentes simplifica a análise, permitindo a utilização de figuras geométricas bidimensionais. Estender as observações a dimensões superiores não incorre em dificuldades extras. Considere que as atenuações promovidas pelo canal e as correlações cruzadas entre as seqüências de espalhamento resultem na matriz [4]:

$$
\mathbf{G}=\left[\begin{array}{ll}
0,5782 & 0,1000 \\
0,2833 & 0,8870
\end{array}\right]
$$

Adicionalmente, para cada usuário $i$, admite-se que a potência do ruído e o índice de QoS sejam estabelecidos por $\sigma_{i}^{2}=0,1 \mathrm{pW}$ e $10 \log _{10}\left(\gamma_{i}^{*}\right)=6 \mathrm{~dB}$, respectivamente. Nestas condições, calcula-se $\mu_{1}(p)$ e $\mu_{2}(p)$, valendo-se da definição dada em (30). A seguir, obtém-se as fronteiras dadas por $\mathcal{L}_{1}(p)=(1+\alpha)\left(2 \alpha \mu_{1}(p)\right)^{-1} \gamma_{1}{ }^{*}$ e $\mathcal{L}_{2}(p)=(1+\alpha)\left(2 \alpha \mu_{2}(p)\right)^{-1} \gamma_{2}{ }^{*}$. Delineia-se a seguir o poliedro $P_{\alpha}$ fazendo-se a intersecção, no primeiro quadrante, da região que satisfaz $p_{1} \leq \mathcal{L}_{1}(p)$ com a que obedece $p_{2} \leq \mathcal{L}_{2}(p)$, conforme apresentado na Fig. 6 .

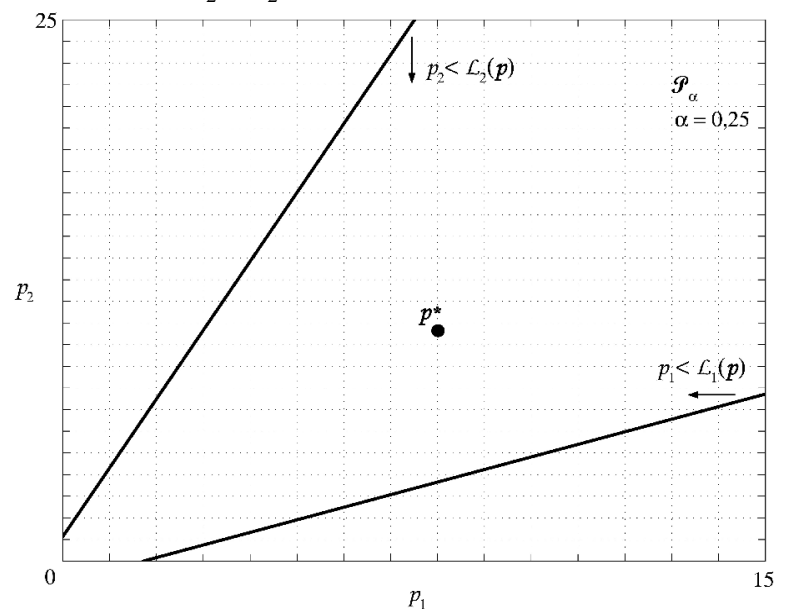

Figura 6. Processo de estruturação do poliedro $P_{\alpha}, \alpha=0,25$.

Note-se que $p_{1}=\mathcal{L}_{1}(p)$ e $p_{2}=\mathcal{L}_{2}(p)$ são simplesmente retas porque o canal reverso está sendo compartilhado por apenas dois usuários. Genericamente, essas igualdades são hiperplanos, conforme citado na Proposição 2. Nessas condições, o cômputo de $(\mathrm{I}-\mathrm{H})^{-1} \eta$ resulta na solução ótima $p^{*}=[8,0110,63]^{\mathrm{T}}$. Por inspeção da Fig. 5, confirma-se que este ponto pertence a $P_{\alpha}$, fato que concorda com a Proposição 3. Para tornar completa a validação desta proposição, deve-se variar o parâmetro $\alpha$ no intervalo $(0,1]$ e verificar se o ponto ótimo localiza-se no interior de cada $P_{\alpha}$ ilustrado na Fig. 7. Observa-se que, apesar do aumento de $\alpha$ aproximar as fronteiras do poliedro, este continua contendo o ponto ótimo $p^{*}$.

$\mathrm{Na}$ Fig. 7, o estreitamento de $P_{\alpha}$ torna maior a possibilidade de se fazer uma seleção não apropriada de condição inicial $p(0)$. Na prática, entretanto, $\alpha$ constitui um parâmetro controlável e, de acordo com o observado na Fig. 6, aproximando-o de zero, pode-se fazer com que as fronteiras englobem o vetor de inicialização. Por outro lado, diminuir $\alpha$ implica em velocidade de convergência menor, pois o mesmo define o passo da integração numérica. 
Portanto, a penalidade por utilizar um valor de $\alpha$ baixo para locar $p(0)$ no poliedro $P_{\alpha}$ consiste na baixa velocidade de convergência resultante. A utilização de um passo $\alpha$ variável constitui uma estratégia para contornar este problema. Neste caso, deve-se incrementar o passo, de um valor suficientemente próximo de zero até a unidade, à medida que as iterações avançam.
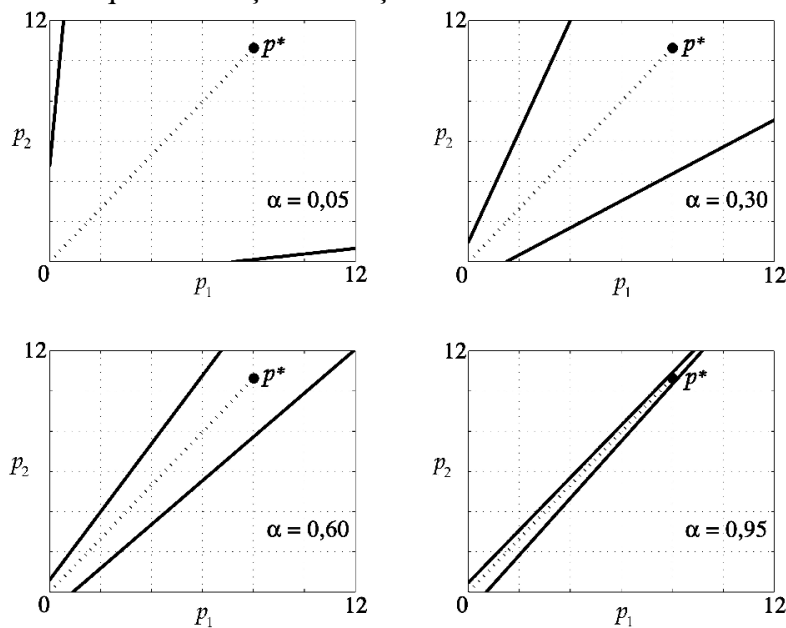

Figura 7. Efeito da variação do parâmetro $\alpha$ sobre a forma do poliedro $P_{\alpha}$.

As semi-retas pontilhadas na Fig. 7 sugerem que todo vetor estritamente positivo e com a mesma direção da solução ótima, mas com menor norma, localiza-se no interior de $P_{\alpha}$ para todo $\left.\left.\alpha \in\right] 0 ; 1\right]$, o que está de acordo com a Proposição 4. Em conseqüência, um vetor com todos os elementos positivos e próximos de zero pertence ao poliedro $P_{\alpha}$ para qualquer $\alpha$ em tal faixa. Portanto, utilizando-se este vetor específico para inicializar o algoritmo proposto, tem-se mais liberdade para fixar o tamanho do passo $\alpha$.

Com o intuito de validar graficamente a Proposição 5, define-se:

$E_{i}(\lambda, p)=\operatorname{sinal}\left\{F_{i}(\lambda p)-\lambda F_{i}(p)\right\}$,

onde $\operatorname{sinal}\{x\}=x / \max \{-x, x\}$.

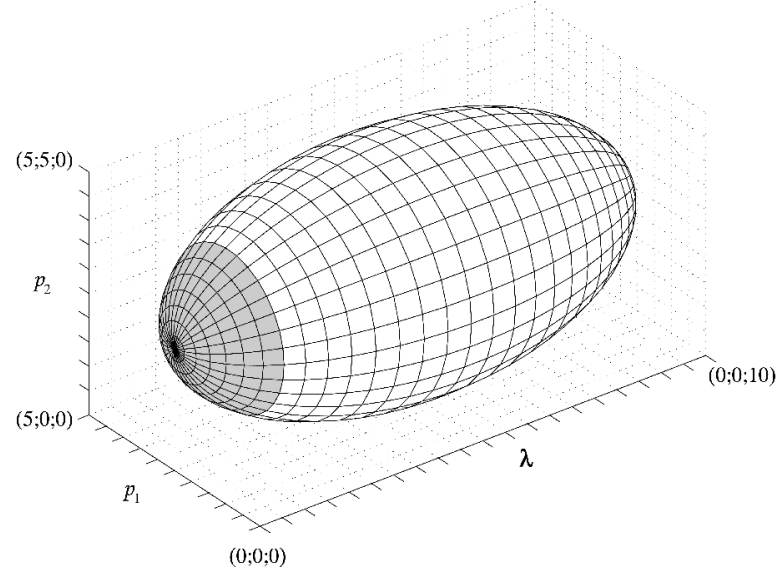

Figura 8. Matização de uma superfície elipsoidal segundo $E_{i}(\lambda, p), i=1,2$.
Considera-se que o sinal de $E_{i}(\lambda, p)$ determina se a região infinitesimal em torno da coordenada $(\lambda, p)$ da superfície arbitrária $\Omega(\lambda, p)=0$ deve ou não receber a cor cinza. Em outros termos, na Fig. 8 , para todo $(\lambda, p)$ condicionado a $\Omega(\lambda, p)=0$ :

$E_{i}(\lambda, p)=+1 \Rightarrow(\lambda, p)$ em cinza

$E_{i}(\lambda, p)=-1 \Rightarrow(\lambda, p)$ em branco

Desta forma, impondo-se que a superfície $\Omega(\lambda, p)=0$ seja elipsoidal, lembrando-se da configuração do cenário e com $\alpha=0,25$, obtém-se a matiz da Fig. 8. Note-se que a "calota" cinza ocorre para $0<\lambda<1$. Este fato, aliado às definições (35) e (36), corrobora a Proposição 5, onde se afirma que $F_{i}(\lambda p)>\lambda F_{i}(p)$ se $0<\lambda<1$ e $\lambda F_{i}(p) \geq F_{i}(\lambda p)$ se $\lambda \geq 1$. Superfícies elipsoidais com diferentes tamanhos fornecem resultado idêntico, no sentido de que sempre se observa uma "calota" cinza na faixa $0<\lambda<1$, confirmando a Proposição 5 de forma ampla.

Sintetiza-se a seguir uma justificativa geométrica para a Proposição 7. Basicamente, analisa-se a relação entre a orientação do deslocamento vetorial $\boldsymbol{d}(p)=\boldsymbol{F}(p)-p$ e a posição do ponto $p$ no interior do poliedro $P_{\alpha}$. Inicialmente, deve ser ressaltado que se atinge a meta de QoS do usuário $i$ quando o vetor $p$ obedecer à equação:

$p_{i}=\gamma_{i}(p)=\gamma_{i}^{*} / \mu_{i}(p)$

Os $K$ hiperplanos deste tipo se encontram na solução ótima $p^{*}$ e seccionam $P_{\alpha}$ de tal forma que se identificam $2^{K}$ regiões distintas quanto à orientação do vetor $\boldsymbol{d}(p)$. Lembrese que no cenário simplificado adotado nesta seção, há apenas dois usuários ocupando ativamente o canal reverso; portanto, existem $2^{K}=4$ dessas regiões, as quais estão denotadas na Fig. 9 por A, B, C e D, onde:

$\mathrm{A}=\left\{p \in P_{\alpha}: p_{1}<\mathscr{Y}_{1}(p)\right.$ e $\left.p_{2}<\mathscr{Y}_{2}(p)\right\}$,

$\mathrm{B}=\left\{p \in P_{\alpha}: p_{1}>\mathscr{Y}_{1}(p)\right.$ e $\left.p_{2}<\mathscr{Y}_{2}(p)\right\}$,

$\mathrm{C}=\left\{p \in P_{\alpha}: p_{1}>\mathscr{Y}_{1}(p)\right.$ e $\left.p_{2}>\mathscr{Y}_{2}(p)\right\}$,

$\mathrm{D}=\left\{p \in P_{\alpha}: p_{1}<\Upsilon_{1}(p)\right.$ e $\left.p_{2}>\Upsilon_{2}(p)\right\}$.

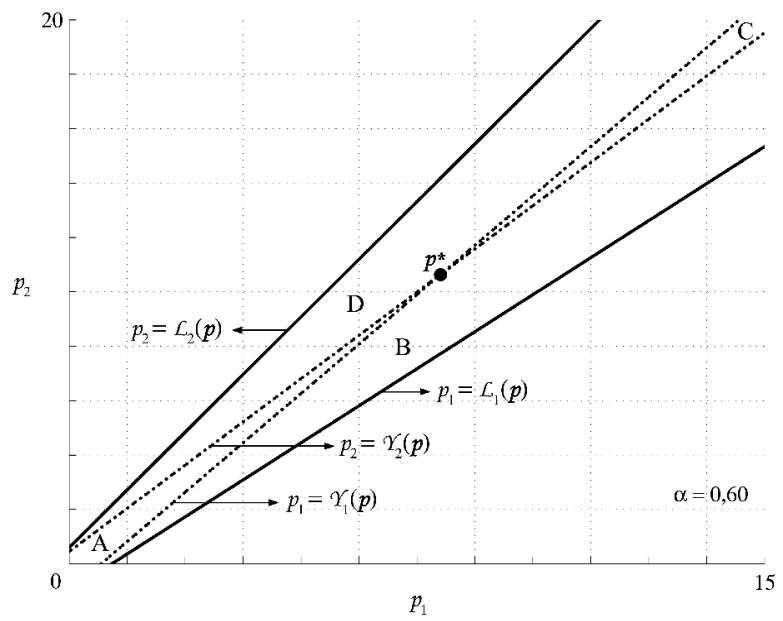

Figura 9. Seccionamento de $P_{\alpha}$ através de $p_{i}=\Upsilon_{i}(p), i=1,2$.

De acordo com (33) e (56), o $i$-ésimo elemento do vetor $\boldsymbol{d}(p)$ vale:

$d_{i}(p)=\alpha p_{i}\left(1-p_{i} / \Upsilon_{i}(p)\right)$ 
Deste modo:

$p_{i}<\mathcal{Y}_{i}(p) \Rightarrow \operatorname{sinal}\left\{d_{i}(p)\right\}=+1$

$p_{i}>\gamma_{i}(p) \Rightarrow \operatorname{sinal}\left\{d_{i}(p)\right\}=-1$

Portanto, (57) equivale a:

$\mathrm{A}=\left\{p \in P_{\alpha}: \operatorname{sinal}\{\boldsymbol{d}(p)\}=[+1+1]^{\mathrm{T}}\right\}$,

$\mathrm{B}=\left\{p \in P_{\alpha}: \operatorname{sinal}\{\boldsymbol{d}(p)\}=[-1+1]^{\mathrm{T}}\right\}$,

$\mathrm{C}=\left\{p \in P_{\alpha}: \operatorname{sinal}\{\boldsymbol{d}(p)\}=[-1-1]^{\mathrm{T}}\right\}$,

$\mathrm{D}=\left\{p \in P_{\alpha}: \operatorname{sinal}\{\boldsymbol{d}(p)\}=[+1-1]^{\mathrm{T}}\right\}$.

As definições em (60) implicam que a região onde se localiza $p$ determina o quadrante do deslocamento $\boldsymbol{d}(p)$. Explicita-se esta correspondência na Fig. 10. Associando a localização de cada região no interior do poliedro (ver Fig. 9) com a respectiva orientação do deslocamento $\boldsymbol{d}(p)$ (ver Fig. 10), infere-se que qualquer ponto em $P_{\alpha}$ tende a permanecer neste ao ser deslocado por $\boldsymbol{F}($.$) . Esta$ propriedade constitui a essência da Proposição 7.

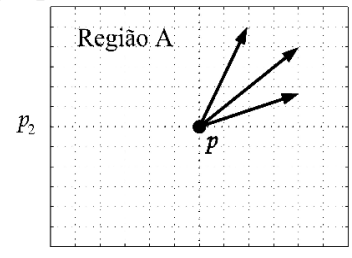

$p_{1}$

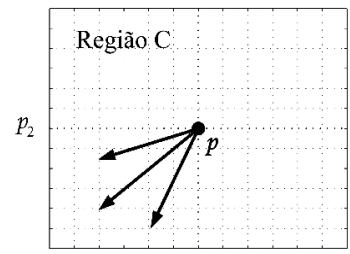

$p_{1}$
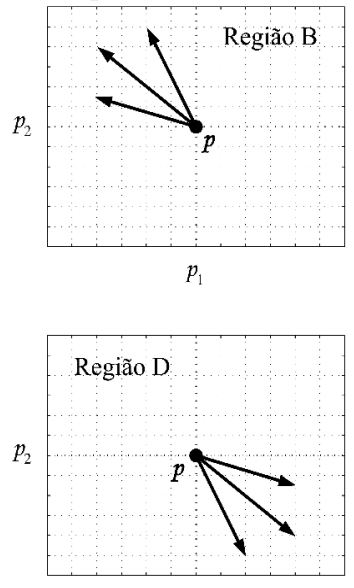

$p_{1}$
Figura 10. Orientação do vetor $\boldsymbol{d}(p)=\boldsymbol{F}(p)-p$ conforme a região de $P_{\alpha}$.

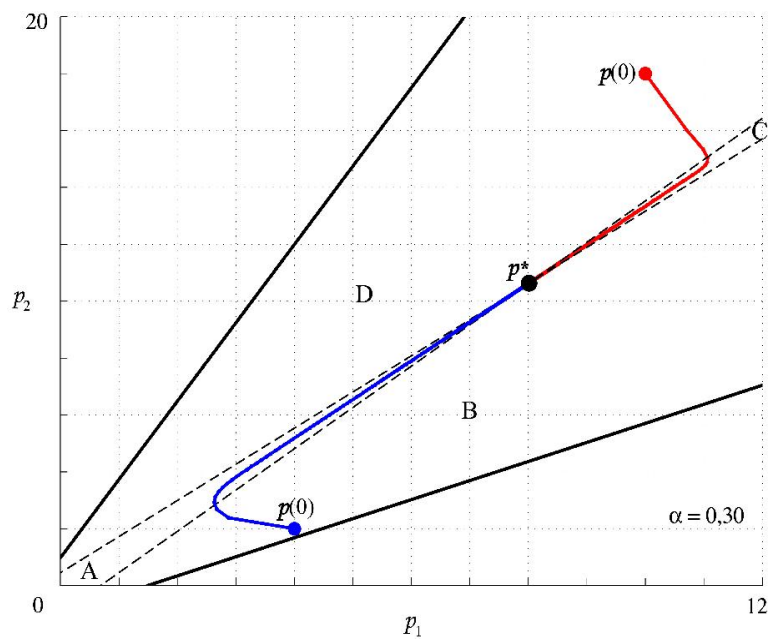

Figura 11. Processos de convergência obtidos por meio de simulação computacional.

$\mathrm{Na}$ Fig. 11 estão indicados dois processos de convergência até $p^{*}$, um iniciado na região $\mathrm{D}$ e outro na região $\mathrm{B}$. Analisando-se o processo iniciado na região $\mathrm{D}$ percebe-se que, durante a permanência na região $\mathrm{D}$, o deslocamento vetorial $\boldsymbol{d}(p)$ limita-se ao quarto quadrante. Entretanto, ao se entrar na região $\mathrm{C}$, sua orientação restringe-se ao terceiro. Analogamente, no processo de convergência iniciado na região $\mathrm{B}$, observa-se $\boldsymbol{d}(p)$ no segundo quadrante, passando para o primeiro ao se atingir a região A. Essas constatações estão coerentes com as asserções expostas na Fig. 10. As descrições em (60) sugerem que se designem as regiões $\mathrm{A}, \mathrm{B}, \mathrm{C}$ e D pelas palavras $11,-11,-1-1$ e $1-1$, respectivamente. Esta notação, além de ser mais simples e compacta, permite que se imagine cada região como um estado transitório de um circuito seqüencial. Neste contexto, especificar um processo de convergência consiste em determinar qual seqüência de estados ocorre até se atingir a solução ótima. Ao todo, distinguem-se $3^{K}$ estados possíveis.

Considere, ainda na Fig. 11, uma circunferência infinitesimal envolvendo $p^{*}$. Percorrendo-a no sentido antihorário, identificam-se os seguintes estados: 11 (A), 01 (fronteira entre A e B), -11 (B), -10 (fronteira entre B e C), -1-1 (C), 0-1 (fronteira entre C e D), 1-1 (D) e 10 (fronteira entre D e A). A solução ótima $p^{*}$ corresponde ao estado 00 e completa a lista de estados possíveis para $K=2$. Os dois processos de convergência, iniciados nas regiões $D$ e $\mathrm{B}, \quad$ equivalem às seqüências $\mathrm{D}=1-1 \rightarrow \mathrm{C}=-1-1 \rightarrow p^{*}=00$ e $\mathrm{B}=-11 \rightarrow \mathrm{A}=11 \rightarrow p^{*}=$ 00 , respectivamente. Note-se que as regiões $\mathrm{A}=11 \mathrm{e}$ $\mathrm{C}=-1-1$ funcionam como "sorvedouros" dos processos de convergência, ou seja, todo procedimento iterativo iniciado no poliedro $P_{\alpha}$ finaliza "capturado" em algum momento por uma dessas duas regiões. No hiperespaço, os estados $111 \ldots 1$ e $-1-1-1 \ldots-1$ constituem os "sorvedouros" das iterações. Estando em 111...1, o vetor de potências aumenta de forma monotônica em direção à solução ótima, denotada pelo estado $000 \ldots 0$. Na região $-1-1-1 \ldots-1$, as potências diminuem monotonicamente de maneira que também se aproximam dos respectivos valores ótimos. Desta forma, o algoritmo proposto realiza a convergência em duas etapas: a) penetra no estado $111 \ldots 1$ ou $-1-1-1 \ldots-1$, tornando (ou mantendo) as potências todas menores ou todas maiores que os limiares necessários às devidas metas de QoS; b) aproxima todas as potências das respectivas quantidades ótimas de forma monotônica. Portanto, pode-se dizer que o algoritmo tende a não privilegiar qualquer parcela de usuários.

Observe-se que o conjunto $m_{\alpha}$, definido durante a demonstração da Proposição 7 , corresponde à região denotada por $111 \ldots 1$. Já a camada $\boldsymbol{C}_{\alpha}$, também caracterizada ao se provar a Proposição 7 , equivale à união das demais regiões que compõem o poliedro $P_{\alpha}$. Ressalte-se que a barreira $\boldsymbol{C}_{\alpha}$ torna-se acentuadamente delgada ao se aproximar $\alpha$ da unidade, pois $\lim _{\alpha \rightarrow+1} \mathcal{L}_{i}(p)=\Upsilon_{i}(p)$. Lembrese que $\gamma_{i}(p)=\gamma_{i}^{*} / \mu_{i}(p)$ e $\mathcal{L}_{i}(p)=(1+\alpha)\left(2 \alpha \mu_{i}(p)\right)^{-1} \gamma_{i}{ }^{*}$.

Visualiza-se na Fig. 12 a evolução das potências transmitidas para três condições iniciais $(\lambda=0,05 ; 0,35$ e 0,70$)$, além da solução ótima $(\lambda=1,00)$. Cada procedimento recursivo apresentado nessa figura principia com um vetor de mesma orientação e menor norma que a solução ótima. Constata-se que as curvas aproximam-se das respectivas assíntotas de maneira 
crescente e monotônica, o que comprova a Proposição 8 para o cenário particular analisado. Observa-se que a taxa de convergência para a condição de equilíbrio experimenta forte influência do parâmetro $\alpha$. Note-se a relação direta: aumento de $\alpha$, acentuação da taxa.
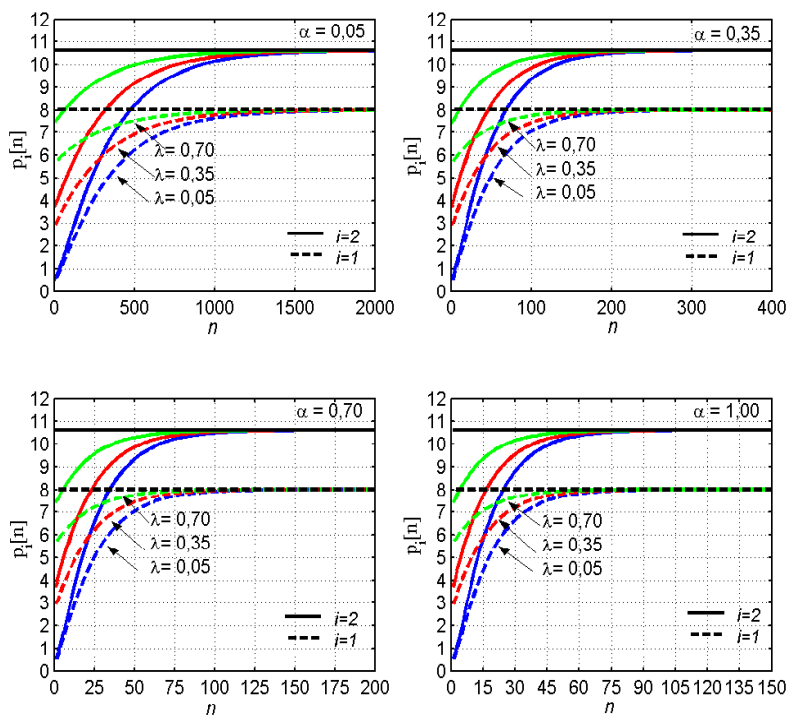

Figura 12. Comportamento típico de $p(n)=\left[p_{1}(n) p_{2}(n)\right]^{\mathrm{T}}$ para $p(0)=\lambda p^{*} \leq p^{*}$.

$\mathrm{Na}$ Eq. (30), verifica-se por substituição que $\boldsymbol{F}(0)=0$. Assim sendo, $p=0$, vetor não pertencente ao poliedro $P_{\alpha}$, define um ponto fixo do método iterativo em questão. Entretanto, pela Proposição 8, a seqüência com termo geral $\boldsymbol{F}^{n}\left(\lambda p^{*}\right)$ converge para $p^{*}$ quando $\lambda$ for ligeiramente superior a zero. Desta forma, a origem constitui ponto de equilíbrio instável. As curvas para $\lambda=0,05$, identificadas pela cor azul na Fig. 12, refletem esta propriedade.
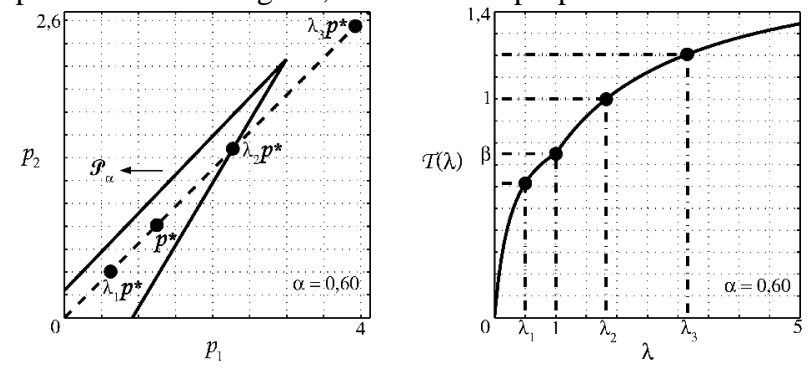

Figura 13. Correspondência entre $\mathcal{T}(\lambda)$ e a posição de $\lambda p^{*}$ relativa a $P_{\alpha}$.

Na síntese da Fig. 13, modificou-se o QoS do segundo usuário para $2 \mathrm{~dB}$, porém manteve-se as demais características do cenário. Essa alteração facilita a percepção visual do vínculo entre a curva $\mathcal{T}(\lambda)$, definida na Proposição 9 , e a posição de $\lambda p^{*}$ em relação ao poliedro $P_{\alpha}$. Na Fig 13, constata-se que a função $\mathcal{T}(\lambda)$ cresce de forma monotônica à medida que $\lambda$ aumenta; no momento que este satisfaz à igualdade $\tau(\lambda)=1$, o ponto $\lambda p^{*}$ correspondente atinge a fronteira de $P_{\alpha}$. Note-se que $\mathcal{T}(\lambda)<1$ e $\mathcal{T}(\lambda)>1$ implicam, respectivamente, $\lambda p^{*}$ interno e externo ao poliedro. Estas observações estão coerentes com a
Proposição 9. Em conseqüência, a equação $\mathcal{T}(\lambda)=1$ constitui um limiar para "detectar" o $\lambda$ crítico, sendo este definido como o maior fator multiplicativo $\lambda$ que não torna $\lambda p^{*}$ externo ao poliedro. Ressalte-se que diferentes cenários resultam, freqüentemente, em distintos fatores críticos. Mas o limiar para identificá-los permanece sendo $\mathcal{T}(\lambda)=1$.

Analisando a Fig. 13, conclui-se que $\mathcal{T}(\beta) \leq 1$ para o cenário em questão. Também se verifica que $\mathcal{T}\left(\beta^{-1}\right) \leq 1$, aliando $\mathcal{T}(\lambda)$ à definição de $\mu_{i}(p)$ em (30). Observe que essas inequações são identidades, no sentido de que não são intrínsecas ao cenário. Nestas argumentações, está implícito que $0<\alpha \leq 1$.

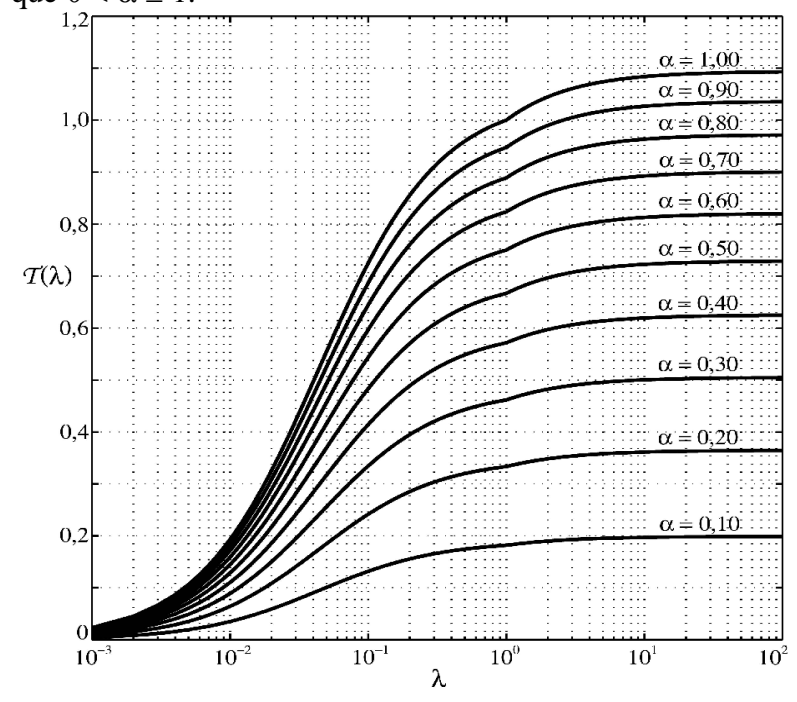

Figura 14. Efeito da perturbação de $\alpha$ na função teste $\mathcal{T}(\lambda)$.

Retornando o QoS do segundo usuário para seu valor anterior $(6 \mathrm{~dB})$, obteve-se a Fig. 14. Note-se que a função teste $\mathcal{T}(\lambda)$ sofre acentuada influência do parâmetro $\alpha$. Valores de $\alpha$ suficientemente próximos a zero fazem com que as respectivas funções teste estejam sempre abaixo do limiar. Esta característica associada aos parágrafos anteriores referentes à Fig. 13 permite afirmar que $o$ poliedro $P_{\alpha}$ tende a englobar completamente a reta $\lambda p^{*}$ conforme $\alpha$ decresce. Essa tendência está subentendida na Fig. 7. Observe-se ainda na Fig. 14 que um $\alpha$ próximo o bastante da unidade faz com que a função teste correspondente intercepte o limiar $\mathcal{T}(\lambda)=1$, o que implica o surgimento de um $\lambda$ crítico, cuja existência significa que $P_{\alpha}$ não contém a reta $\lambda p^{*}$ completamente, coerente com a Fig. 7 , onde se observa o fechamento de $P_{\alpha}$ à medida que $\alpha$ é incrementado em direção à unidade. Ainda na Fig. 14, verifica-se que $T(\lambda) \leq 1$ quando $0<\lambda \leq 1$ e $0<\alpha \leq 1$, fato coerente com a Proposição 4.

Na Fig. 15, para um dado $\alpha$, todo processo iterativo, cada qual identificado por um $\lambda$ distinto, inicia com um vetor proporcional à solução ótima, mas com maior norma, e locado no interior do poliedro $P_{\alpha}$; estão incluídas as curvas referente à solução ótima. As curvas referentes a cada processo recursivo atingem as respectivas assíntotas de forma decrescente e monotônica, o que corrobora a Proposição 10. Para garantir que $p(0)=\lambda p^{*} \in P_{\alpha}$, cada $\alpha$ 
usado na síntese da Fig. 15 corresponde a uma curva $\mathcal{T}(\lambda)$ na Fig. 14 menor que a unidade na faixa $0<\lambda \leq 2,5$. Adicionalmente, a velocidade com que ocorre a estabilização das iterações aumenta acentuadamente conforme o passo $\alpha$ cresce em direção à unidade. Além disso, para um dado $\alpha$, constata-se que todos os processos iterativos tornam-se visualmente indistingüíveis das assíntotas aproximadamente na mesma iteração. Na Fig. 12, identifica-se esta mesma propriedade. Desta forma, o intervalo de tempo necessário para se atingir o estado estacionário não sofre expressiva influência da condição inicial, pelo menos quando esta é proporcional ao vetor ótimo.
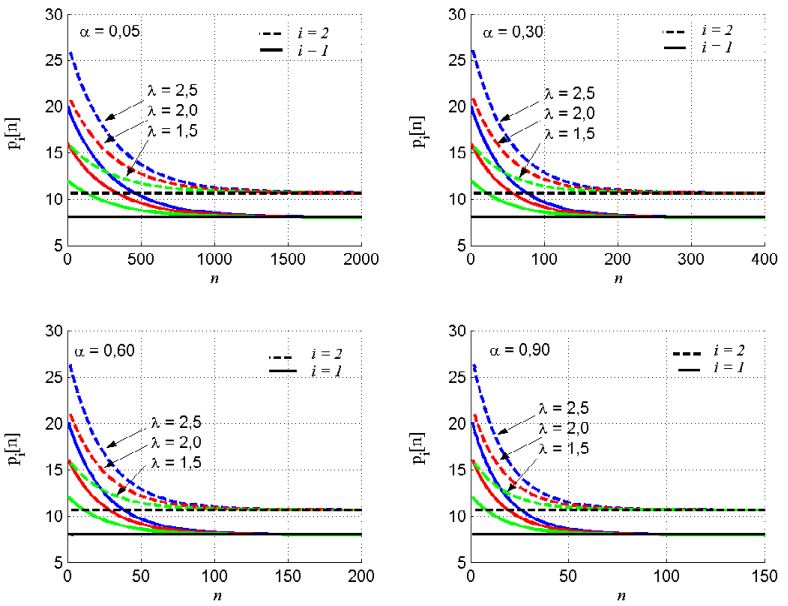

Figura 15. Comportamento típico de $p(n)=\left[p_{1}(n) p_{2}(n)\right]^{\mathrm{T}}$ para $p(0)=\lambda p^{*} \geq p^{*}$.
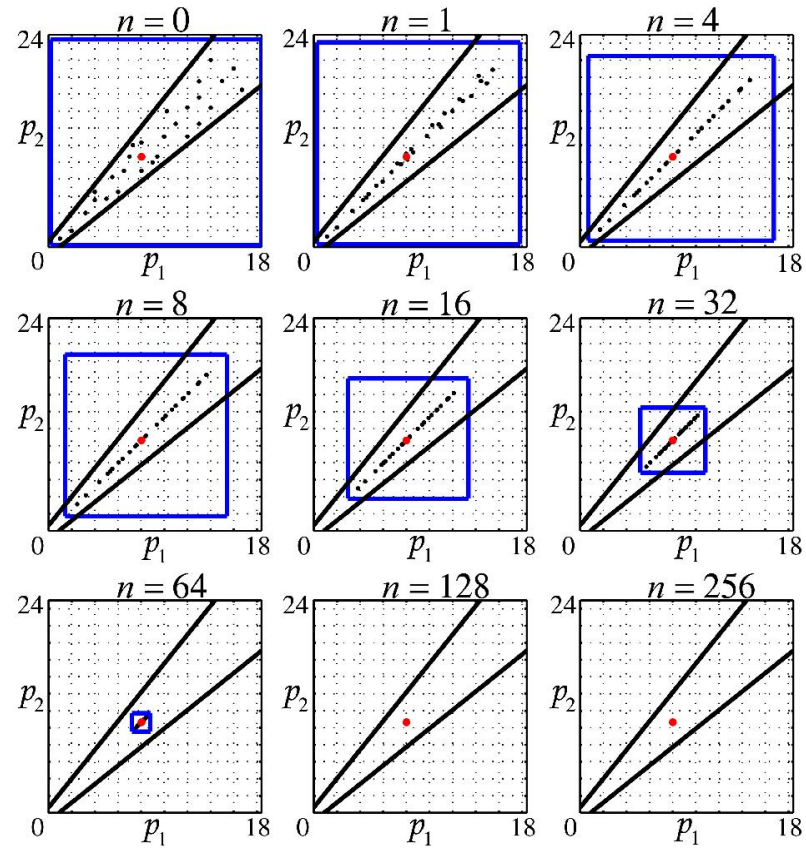

Figura 16. Configuração dos pontos conforme o número de iterações, $K=2$ e $\alpha=0,6$.

A evolução de uma coleção de pontos conforme se aplica $\boldsymbol{F}$ (.) a cada um destes é retratada na Fig. 16. Parte-se de um volume $\mathbf{J}$ de vetores iniciais distribuídos uniformemente no interior de $P_{\alpha}$. Considere o problema de determinar uma "hipercaixa" que contenha todas as condições iniciais e adicionalmente possua dois de seus vértices proporcionais ao ponto ótimo e locados no poliedro $P_{\alpha}$. Resolve-se esta questão encontrando-se $\lambda_{1}$ e $\lambda_{2}$ de forma que, para $j=1,2$, ..., J:

$\lambda_{1} p^{*} \leq p^{j}(0) \leq \lambda_{2} p^{*}$

onde $p^{j}(0)=\left[p_{1}^{j}(0) \cdots p_{i}^{j}(0) \cdots p_{K}^{j}(0)\right]^{\mathrm{T}}$ é o vetor inicial $j$.

Para tanto, calcula-se primeiramente:

$\lambda(i, j)=p_{i}^{j}(0) / p_{i}^{*}$, em todos os $K \times \mathrm{J}$ pares $(i, j)$

Em seguida, dentre os valores obtidos, separa-se o ínfimo e o supremo:

$\lambda_{\text {inf }}=\inf \{\lambda(i, j)\}$ e $\lambda_{\text {sup }}=\sup \{\lambda(i, j)\}$

Finalmente, deve-se selecionar $\lambda_{1} \leq \lambda_{\text {inf }}$ e $\lambda_{2} \geq \lambda_{\text {sup. }}$. Adicionalmente, a constante $\lambda_{2}$ deve ser tal que $\tau\left(\lambda_{2}\right) \leq 1$, pois deseja-se que $\lambda_{1} p^{*}$ e $\lambda_{2} p^{*}$ estejam em $P_{\alpha}$.

Uma vez que os resultados numéricos da Fig. 16 referemse a um cenário bidimensional, a "hipercaixa" estruturada de acordo com os passos anteriores reduz-se ao retângulo visualizado nessa figura em $n=0$. Os retângulos apresentados na Fig. 16 variam $\operatorname{com} n$, pois são definidos pelos vértices $\boldsymbol{F}^{n}\left(\lambda_{1} p^{*}\right)$ e $\boldsymbol{F}^{n}\left(\lambda_{2} p^{*}\right)$. Ainda nesta figura, todos os pontos permanecerão no interior do retângulo à medida que as iterações prosseguirem. Desta forma, para todo $n$ :

$\boldsymbol{F}^{n}\left(\lambda_{1} p^{*}\right) \leq \boldsymbol{F}^{n}\left(p^{j}(0)\right) \leq \boldsymbol{F}^{n}\left(\lambda_{2} p^{*}\right), j=1,2, \ldots, \mathbf{J}$

As dimensões do retângulo colapsam com o aumento de $n$. Sua superfície, contendo os $\mathrm{J}$ pontos, tende a se degenerar em torno da solução ótima. Na escala da Fig. 16, já para $n=128$ iterações, não há possibilidade de distinguir visualmente o ponto ótimo do retângulo. Assim, infere-se que:

$\lim _{n \rightarrow \infty} \boldsymbol{F}^{n}\left(\lambda_{1} p^{*}\right)=\lim _{n \rightarrow \infty} \boldsymbol{F}^{n}\left(\lambda_{2} p^{*}\right)=$

$=\lim _{n \rightarrow \infty} \boldsymbol{F}^{n}\left(p^{j}(0)\right)=p^{*}, j=1,2, \ldots, \mathbf{J}$

Os resultados em (64) e (65), obtidos numericamente, equivalem, respectivamente, às equações (49) e (50), as quais constituem a essência da Proposição 11. Destaca-se que os $\mathrm{J}$ pontos, após poucas iterações, alinham-se e cada qual passa a localizar-se ou na região $111 \ldots 1$ ou na $-1-1-1 \ldots-1$ do poliedro $P_{\alpha}$ ("fenômeno sorvedouro"). Percebe-se que os pontos organizam-se de tal modo que originam uma configuração espacial no interior do retângulo que, a menos de um fator de escala, repete-se indefinidamente com o incremento das iterações. Em outras palavras, após o alinhamento, ampliando-se convenientemente o retângulo em $n+m$, consegue-se o aspecto em $n$. Para se certificar disto, basta observar na Fig. 16 as configurações nos instantes $n=8$ e $n=16$.

O comportamento do algoritmo proposto é analisado admitindo-se agora interferência constante. Impondo-se $\xi_{i}(p)=\gamma_{i}^{*} / \mu_{i}(p)=\xi=$ constante em (30), desacopla-se os usuários, e a análise da convergência reduz-se à seguinte iteração unidimensional:

$p(n+1)=F(p(n))=(1+\alpha) p(n)-\alpha \xi^{-1} p(n)^{2}$

Esta recursão, simples na forma, possui ampla diversidade de comportamentos conforme se modifica $\alpha$. Considere os intervalos $I_{\alpha}=\left(0, \xi(1+\alpha)^{+2}(4 \alpha)^{-1}\right] \quad$ e $I^{\prime}{ }_{\alpha}=\left(0, \xi(1+\alpha) \alpha^{-1}\right]$. A parábola côncava $F(p)$ em (66) 
produz valores em $I_{\alpha}$ quando $p$ estiver em $I^{\prime}{ }_{\alpha}$. Não estando $p$ neste intervalo, obtém-se $F(p) \leq 0$. Portanto, deduz-se que $p(0)$ deve estar necessariamente no intervalo $I_{\alpha}^{\prime}$ e que a seqüência $p(n+1)=F(p(n))$, ao atingir o regime permanente, fica confinada em $I_{\alpha}$.

Nas asserções acima, está implícito que $I_{\alpha} \subset I^{\prime}{ }_{\alpha}$. Caso contrário, em alguma iteração $n$, tem-se $F(p(n)) \leq 0$, o que faz o algoritmo divergir. Evidentemente, a propriedade $I_{\alpha} \subset I^{\prime}{ }_{\alpha}$ não é válida para $\alpha>3$. Portanto, basta analisar o comportamento de (66) para $\alpha$ na faixa $0<\alpha \leq 3$.

\section{RESULTADOS NUMÉRICOS}

Esta seção traz uma análise comparativa dos algoritmos clássico [1] e sigmoidal [4] com o proposto em termos das principais características de um algoritmo de controle de potência distribuído: a) o quão próxima da solução do problema de alocação está a solução encontrada pelo algoritmo; b) consumo médio de potência por usuário quando o algoritmo atinge o regime permanente; c) número de atualizações para o algoritmo atingir a condição de regime permanente; d) complexidade de implementação.

A característica a) pode ser quantificada por meio do erro quadrático médio normalizado (NSE) na condição de regime permanente. A definição do NSE é dada por [5]:

$\operatorname{NSE}(n)=E\left[\frac{\left\|\mathbf{p}(n)-\mathbf{p}^{*}\right\|^{2}}{\left\|\mathbf{p}^{*}\right\|^{2}}\right]$

Onde $\|\cdot\|^{2}$ denota o quadrado da distância euclidiana em relação à origem e $E[\mathrm{~g}]$ o operador esperança.

O consumo médio de potência por usuário na $n$-ésima iteração é calculado por:

$\operatorname{PM}(n)=E\left[\frac{1}{K} \sum_{i=1}^{K} p_{i}(n)\right]$

$\mathrm{O}$ valor de (68) quando o algoritmo atinge o regime permanente quantifica a característica $b$ ). O número de iterações necessárias para o $\operatorname{NSE}(n)$ ficar confinado em um intervalo estreito pré-estabelecido quantifica a característica c). Finalmente, uma forma simples de quantificar a característica d) consiste em obter o total de somas, subtrações, divisões, multiplicações e acessos a valores em tabelas necessários à realização de uma atualização do algoritmo multiplicado pelo número de atualizações necessárias para a convergência, $m$.

\subsection{PARÂMETROS DE SIMULAÇÃO}

As simulações foram realizadas em ambiente Matlab 6.5 para $K=5,10$ e 15 usuários em um sistema DS/CDMA. Admite-se que a seguinte condição seja sempre satisfeita:

$m L T_{b}<<(\Delta t)_{c}$

Onde $T_{b}$ e $(\Delta t)_{c}$ são, respectivamente, o período de bit de informação e o tempo de coerência do canal ${ }^{1}$. Esta condição faz parte do processo de estimação da SINR, implicando que cada atualização de potência realizada pelo DPCA

\footnotetext{
${ }^{1}$ Corresponde ao intervalo de tempo no qual as características do canal não experimentam variação expressiva.
}

ocorre em intervalos de pelo menos $L$ bits. As recursões em (20), (25) ou em (31) devem convergir para a solução ótima do problema de alocação antes que cada ganho de canal $g_{i i}$ experimente significativa alteração ${ }^{2}$.

Note-se que satisfeita (69), as matrizes de ganhos permanecem estáticas no intervalo de tempo referente a um único processo de convergência. Nas simulações realizadas, os elementos de cada matriz $\mathbf{G}$ possuem valores intermediários entre os usados em [4, eq. (26)] e [9, Ex. I]. As metas de QoS utilizadas estão entre $4 \mathrm{~dB}$ e $6 \mathrm{~dB}$, faixa baseada em [4] e [5]. A potência de ruído no receptor é $\sigma_{i}^{2}=0,1 \mathrm{pW}, i=1, \mathrm{~L}, K$, para todos os usuários, valor também usado em [4, Ex. 1].

$\mathrm{O}$ algoritmo proposto e o de Foschini et al. foram simulados para $\alpha^{\prime}$ s (passos de integração numérica) positivos não maiores do que a unidade, pois ambos divergem para outros valores de passo [1]. De acordo com [4], o sigmoidal converge para qualquer $\alpha$ positivo. Entretanto, foram adotados $\alpha$ 's não maiores do que 1 para o mesmo devido à excessiva degradação do desempenho em termos de proximidade da solução ótima em regime permanente à medida que $\alpha$ aumenta além da unidade.

$\mathrm{Na}$ prática, as estimativas das SINR's nas estações rádio base não são perfeitas, no sentido de que os valores obtidos no processo de estimação continuam possuindo caráter aleatório. Para incorporar esta situação realista às simulações, adicionou-se um erro aleatório às SINR's calculadas a cada iteração. Considerou-se que a razão entre o valor estimado da SINR e o verdadeiro vale $(1+\varepsilon)$, onde $\varepsilon$ é uma variável aleatória uniformemente distribuída no $[-\delta ;+\delta]$. Realizaram-se as simulações para os seguintes $\delta$ 's: 0,$01 ; 0,05 ; 0,1 ; 0,2 ;$ e 0,25 . Estes valores também foram utilizados em [4, Exemplo 1].

Em todas as simulações, os três algoritmos iterativos foram inicializados com o vetor de potências $\mathbf{p}(0)=[0,1$; $0,1 ; \ldots ; 0,1]^{\mathrm{T}} \mathrm{pW}$, o qual localiza-se no poliedro que delimita a região de monotonicidade do algoritmo proposto (Seção 6).

\subsection{RESULTADOS DE SIMULAÇÃO}

Em seguida, o comportamento dos três algoritmos foi analisado para $K=5,10$ e 15 usuários, obtendo-se a curva $\operatorname{NSE}(n)$, eq. (67), a partir da geração de 1000 funções amostra $\mathbf{p}(n)$ para cada algoritmo, cada qual executando 2000 iterações. Sintetizou-se a curva $\operatorname{PM}(n)$, eq. (68), de cada algoritmo de forma análoga. Obteve-se então uma média com os últimos 500 pontos de cada curva $\operatorname{NSE}(n)$ e $\operatorname{PM}(n)$. Estes valores representam, respectivamente, o NSE e o PM em regime permanente. Finalmente, com esses valores em regime permanente calcularam-se as seguintes razões:

$$
\mathrm{NSEG}_{\text {Foschini }}=\frac{\mathrm{NSE}_{\text {Proposto }}^{\text {regime }}}{\mathrm{NSE}_{\text {Foschini }}^{\text {regime }}}
$$

\footnotetext{
${ }^{2}$ A seleção de um L apropriado é fundamental. Se for escolhido um valor pequeno, as atualizações de potência serão mais freqüentes, o que faz o algoritmo convergir mais rapidamente. Entretanto, essas realimentações freqüentes no canal direto diminuem a capacidade do sistema, pois "consomem" banda.
} 


$$
\begin{aligned}
\mathrm{NSEG}_{\text {Sigmoidal }} & =\frac{\mathrm{NSE}_{\text {Proposto }}^{\text {regime }}}{\mathrm{NSE}_{\text {Sigmoidal }}^{\text {regime }}} \\
\mathrm{PMG}_{\text {Foschini }}= & \frac{\mathrm{PM}_{\text {Proposto }}^{\text {regime }}}{\mathrm{PM}_{\text {Foschini }}^{\text {regime }}} \\
\mathrm{PMG}_{\text {Sigmoidal }} & =\frac{\mathrm{PM}_{\text {Proposto }}^{\text {regime }}}{\mathrm{PM}_{\text {Sigmoidal }}^{\text {regime }}}
\end{aligned}
$$

Repetiu-se o procedimento descrito acima para conjuntos $(\alpha, \delta, K)$ representativos. Os resultados estão mostrados nas Figs. 18 a 23. Para cada $(\alpha, \delta, K)$ existem três curvas $\operatorname{NSE}(n)$, cada qual referente a um algoritmo. Considere o máximo desvio, nos últimos 500 pontos, de cada uma dessas três curvas em relação aos respectivos valores de regime permanente ${ }^{3}$. Dentre esses três valores máximos, selecionou-se o maior, que em seguida foi multiplicado por 2. O valor resultante deste procedimento define o máximo desvio que cada uma das três curvas $\operatorname{NSE}(n)$ pode ter em regime permanente. Assim, o número de iterações necessárias para a convergência de cada algoritmo foi definido como aquele a partir do qual o desvio da correspondente curva $\operatorname{NSE}(n)$ em relação ao seu valor de regime permanente é sempre menor que esse máximo desvio. Note-se que cada tripla $(\alpha, \delta, K)$ resulta em um desvio máximo diferente.

Assim, o número de iterações necessário para a convergência de cada algoritmo depende de $(\alpha, \delta, K)$. Entretanto, o parâmetro determinante da velocidade de cada algoritmo é $\alpha$, pois constitui o próprio passo de integração numérica. Para cada $\alpha$, realizou-se a média em $\delta$ e em seguida em $K$. Os resultados obtidos são mostrados nas curvas da Fig. 17. Denota-se $m_{\text {avg }}$ o número médio de iterações para a convergência de cada algoritmo.

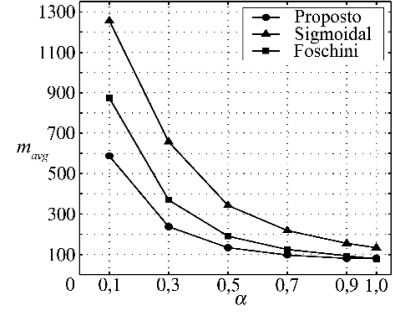

(a)

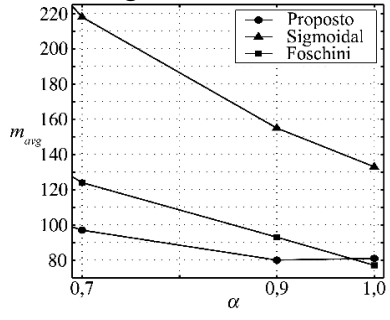

(b)
Figura 17. Número médio de iterações para a convergência em função de $\alpha$.

Constata-se na Fig. 17 que o algoritmo proposto possui desempenho superior em termos de número médio de iterações em relação aos outros dois, exceto para $\alpha=1$, situação em que o de Foschini et al. mostra-se superior aos demais, porém de forma marginal em relação ao proposto. Observe-se que a discrepância de ambos em relação ao proposto é maior conforme $\alpha$ decresce. Existindo um limite

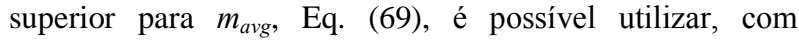
vantagem, um passo $\alpha$ menor para o proposto, o que é importante, pois sabe-se que menores passos de integração numérica resultam em maior proximidade, em regime permanente, da solução ótima.

${ }^{3}$ Ressalte-se que a média nos últimos 500 pontos foi adotada como representante do valor de regime permanente.

72
Conforme as Figs. 19, 21 e 23, o DPCA proposto, para qualquer $\alpha$ e $\delta$, resulta em menor consumo de potência por usuário (PM em regime permanente) em relação aos outros dois. Observa-se que essa vantagem em termos de PM em regime permanente tende a ser maior à medida que o erro de estimação e o passo $\alpha$ aumentam. Essa economia de potência por parte do algoritmo proposto constitui efetivamente uma vantagem se o vetor de potências em regime permanente fornecido pelo mesmo não ficar muito distante (alto NSE em regime permanente) do vetor ótimo. Já os resultados nas Figs. 18, 20 e 22 comprovam que na maioria das situações o algoritmo proposto atinge um menor NSE em regime permanente; nas poucas situações restantes, o NSE em regime permanente é comparável aos valores de NSE em regime permanente dos outros dois DPCA's.
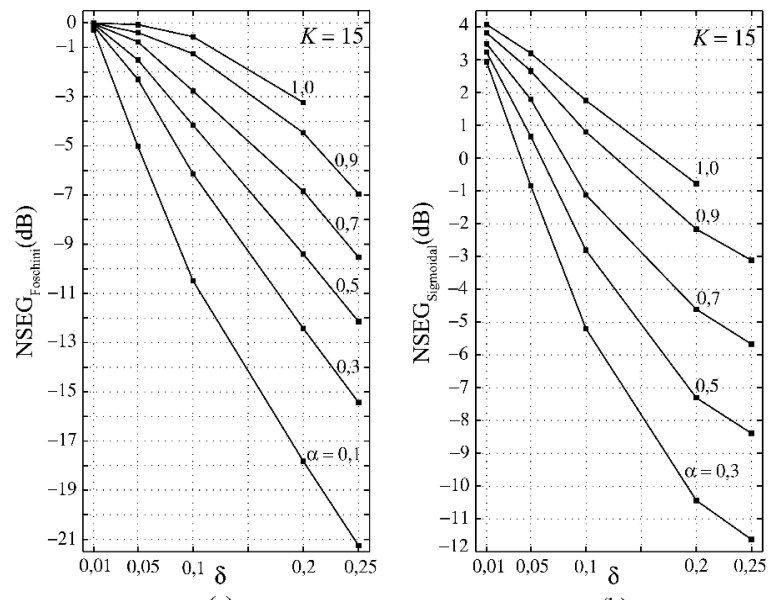

(b)

Figura 18. NSEG em regime permanente para $K=15$.

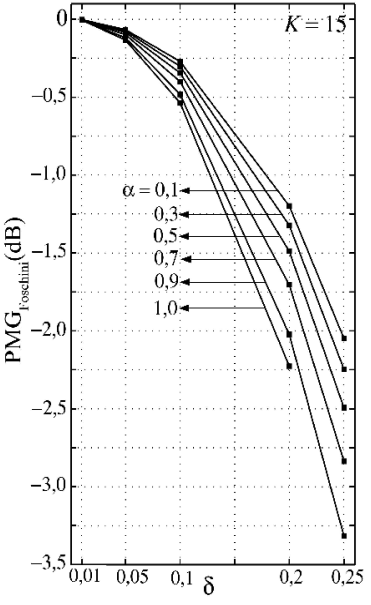

(a)

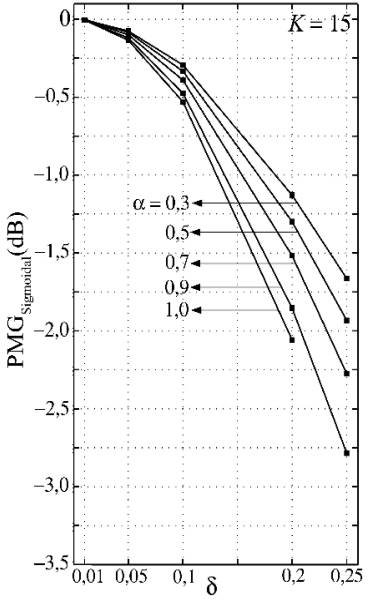

(b)
Figura 19. $\mathrm{PMG}$ em regime permanente para $K=15$.

Constata-se que o NSEG Sigmoidal $_{(\mathrm{dB})}$ apresenta uma tendência positiva ao se diminuir $\delta$, tendendo a superar o algoritmo proposto em termos de NSE em regime permanente apenas para baixos erros de estimação. Observe-se que o quão baixo deve ser o $\delta$ para que $\mathrm{NSEG}_{\text {Sigmoidal }}(\mathrm{dB})$ torne-se positivo depende fortemente de

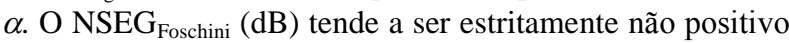
quando o sistema está carregado com mais de 2 usuários. 
Para $K>2$, a vantagem do proposto em relação ao de Foschini et al em termos de NSE em regime permanente aumenta estritamente com $\delta$. Constata-se que a diminuição do passo $\alpha$ tende a beneficiar mais o DPCA proposto que os outros dois, em termos de NSE em regime permanente.

As curvas $\mathrm{NSEG}_{\text {Sigmoidal }}(\mathrm{dB})$ e $\mathrm{PMG}_{\text {Sigmoidal }}(\mathrm{dB})$ quando $\alpha=0,1$ não foram incluídas nas Figs. 18 a 22, pois 0 algoritmo sigmoidal não atingiu a situação de regime permanente após 2000 iterações. A diminuição da quantidade de pontos nessas Figs à medida que o número de usuários decresce (baixos carregamentos) deve-se à tendência do algoritmo proposto não convergir quando há, simultaneamente, baixo carregamento, elevado erro de estimação e passo $\alpha$ próximo da unidade.

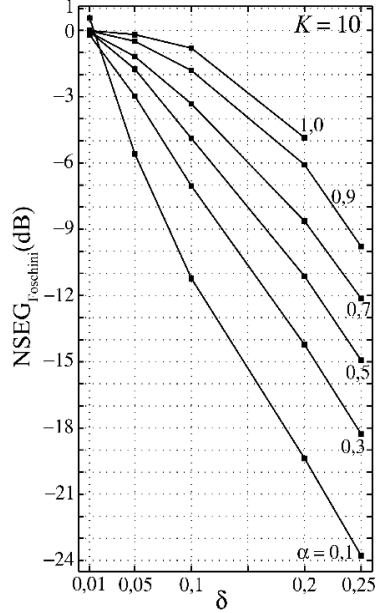

(a)

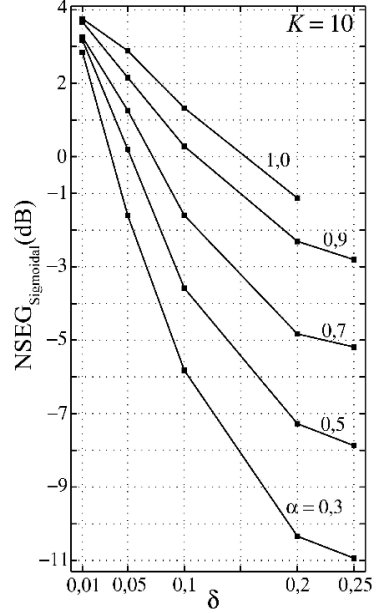

(b)
Figura 20. NSEG em regime permanente para $K=10$.

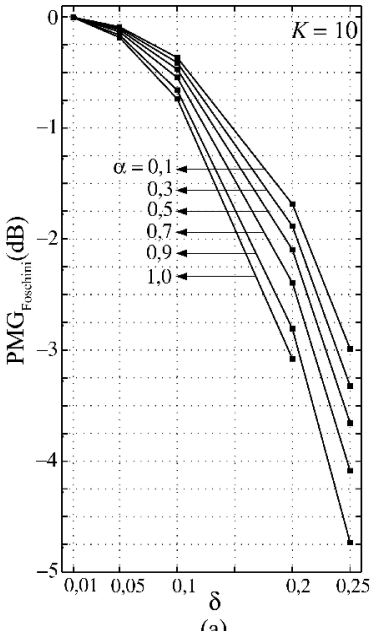

(a)

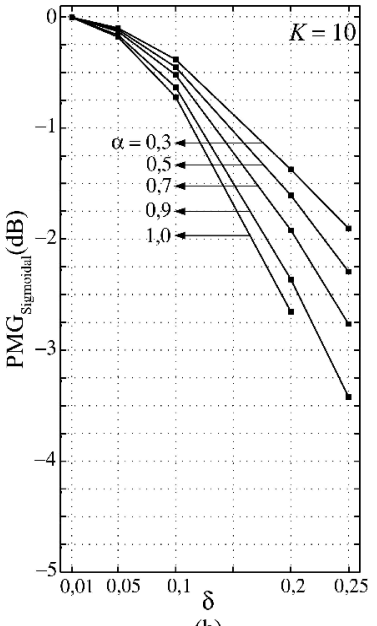

(b)
Figura 21. PMG em regime permanente para $K=10$.

No que tange à complexidade, como no método distribuído cada elo usuário-estação rádio base, $M_{i} B_{i}$, realiza isoladamente suas atualizações, ou seja, o controle de potência como um todo é realizado por $K$ processadores em paralelo e cada qual executa apenas operações escalares, a análise reduz-se ao estudo de uma iteração. Conclui-se que uma iteração no DPCA proposto e no de Foschini et al. possuem a mesma complexidade. Já uma iteração no sigmoidal exige, adicionalmente, acesso a uma tabela para o cômputo da função tanh(.); portanto, o DPCA sigmoidal possui maior complexidade de implementação.

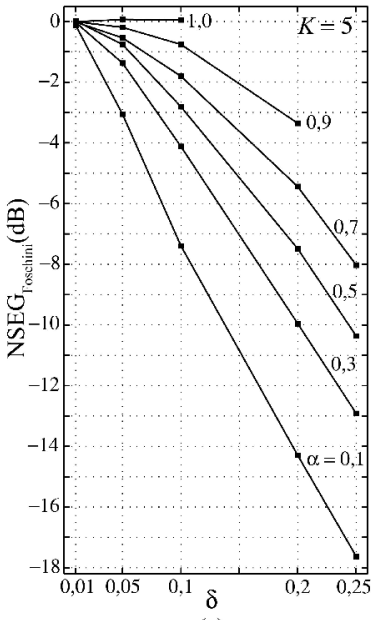

(a)

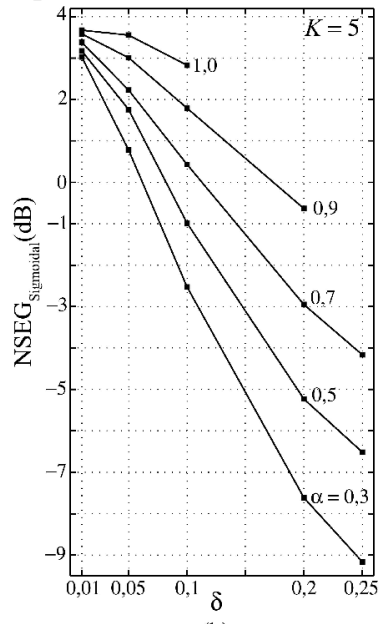

(b)
Figura 22. NSEG em regime permanente para $K=5$.
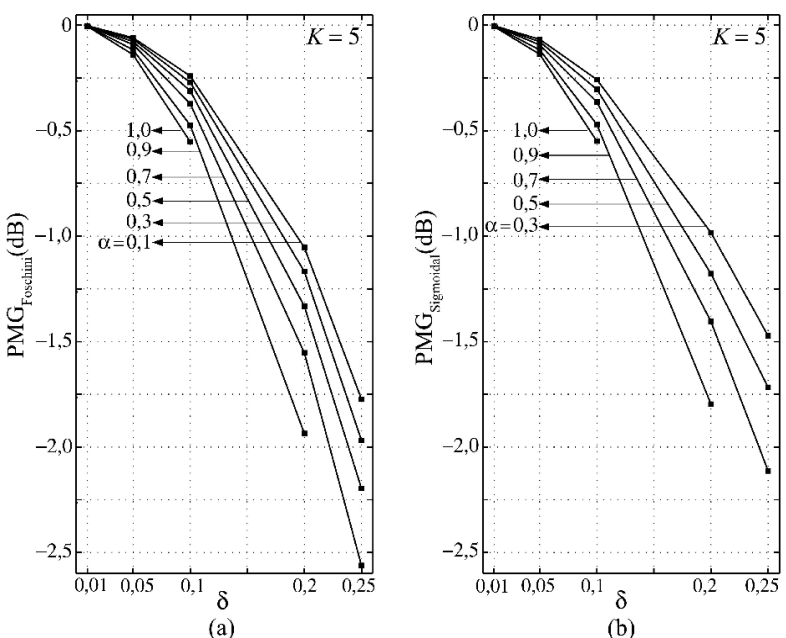

Figura 23. PMG em regime permanente para $K=5$.

\section{CONCLUSÕES}

Apresentou-se e analisou-se um novo algoritmo DPCA para sistemas CDMA em termos das métricas adotadas na comparação de desempenho. O DPCA proposto mostrou-se superior a dois algoritmos bastante conhecidos na literatura em situações práticas de interesse, isto é, quando ocorrerem erros nas estimativas de $\gamma_{i}$ associados a sistemas mediana e altamente carregados.

O Teorema dos GC aliado à estrutura matricial particular do problema de alocação resultou na definição de um conjunto de critérios suficientes para a viabilidade. Mostrou-se que todo sistema com norma-s, $\mathrm{s}=1$, de cada vetor-linha de $\mathbf{H}$ ou $\mathbf{H}^{\mathbf{T}}$ menor do que a unidade é factível. Verificar estas condições exige pouco esforço computacional em relação ao necessário para a estimação efetiva do autovalor de Perron-Frobenius. Adicionalmente, a metodologia sintetizada permite avaliar de uma forma simples a tendência sobre a viabilidade conforme sejam modificados os parâmetros que definem $h_{i j}$, ou seja, a 
atenuação provocada pelo canal sobre o sinal transmitido $j$, a correlação cruzada entre as seqüências de espalhamento $i$ e $j$, e o nível de QoS designado ao usuário $j$.

O diagrama de controle da Fig. 1 é geral e constitui uma forma sistemática de descrever o comportamento dinâmico dos diversos DPCA's. Observe que os três algoritmos analisados neste artigo são estruturas particulares do sistema da Fig. 1. Esta forma de descrição genérica pode facilitar eventuais comparações e o processo de síntese de novos algoritmos distribuídos.

Os algoritmos distribuídos simulados na ausência de erro de estimação apresentaram resultados que na prática podem ser considerados idênticos. Entretanto, quando as medidas das SINR's não foram consideradas perfeitas, as diferenças entre os DPCA's mostraram-se substanciais. O DPCA proposto alcançou a condição de regime permanente mais rapidamente e de forma assintótica. Adicionalmente, o DPCA proposto atingiu menor distância euclidiana do ponto $K$-dimensional ótimo quando as velocidades de convergência dos três algoritmos mostraram-se próximas. Em suma, o DPCA proposto resultou em pronunciada robustez aos erros de estimação aleatórios quando comparado aos algoritmos clássico e sigmoidal.

\section{REFERÊNCIAS}

[1] G. Foschini e Z. Miljanic, "A Simple Distributed Autonomous Power Control Algorithm and its Convergence", IEEE Trans. Veh. Technol., Vol. 42, Nov. 1993.

[2] Verhulst, P. F., (1838). Notice sur la loi que la population pursuit dans son accroissement. Corresp. Math. Phys. 10:113121.

[3] Carl D. Meyer, Matrix Analysis and Applied Linear Algebra, SIAM Publications, 2000.

[4] Z. Uykan e H. Koivo, "Sigmoid-Basis Nonlinear PowerControl Algorithm for Mobile Radio Systems", IEEE Trans. Veh. Technol., Vol. 53, Jan. 2004.

[5] S. Ulukus and R. D. Yates, "Stochastic Power Control for Cellular Radio Systems”, IEEE Trans. On Commun., Vol. 46, No. 6, June 1998.

[6] E. Seneta, Non-Negative Matrices and Markov Chains, 2nd ed. New York: Springer-Verlag, 1981.

[7] B. Sklar, "Rayleigh Fading Channels in Mobile Digital Communication Systems, Part I: Characterization", IEEE Commun. Magazine, July 1997.

[8] W. H. Press, S. A. Teukolsky, W.T. Vetterling, Brian P. Flannery, Numerical Recipes in C: The Art of Scientific Computing, 2nd ed., Cambridge: Cambridge University Press, 1992.

[9] C. C. Chai et al. "A Unified Framework for Transmitter Power Control in Cellular Radio Systems", ETRI Journal, Vol. 26, No. 5, pages 423-31, October 2004.

[10]R. D. Yates. "A Framework for Uplink Power Control in Cellular Radio Systems", IEEE Journal on Selected Areas in Communications, Vol. 13, No. 7, pages 1341-47, September 1995.

Tadeu Júnior Gross é graduado em Engenharia Elétrica pela Universidade Estadual de Londrina em 2.002. Obteve em 2.005 o título de Mestre em Engenharia Elétrica pela Escola Politécnica da Universidade de São Paulo. Atualmente trabalha como Engenheiro Eletricista da Área Instrumental da Secretaria de Administração do Estado do Mato Grosso, Professor das Faculdades Unicen-MT e Pesquisador na área de análise e síntese de algoritmos distribuídos de controle de potência aplicáveis em sistemas CDMA.
Taufik Abrão é graduado, Mestre e Doutor em Engenharia Elétrica em 1992, 1996 e 2001, respectivamente, pela Escola Politécnica da Universidade de São Paulo. Desde 1997 é docente do Departamento de Engenharia Elétrica da Universidade Estadual de Londrina, PR. Atualmente é professor adjunto AD-C. Suas áreas de interesse incluem comunicações sem fio, projeto de sequiências de códigos para uso em sistemas DS-CDMA, detecção multiusuário e estimação de parâmetros em DS-CDMA, técnicas heurísticas aplicáveis a problemas de otimização em sistemas de múltiplo acesso.

Paul Jean Etienne Jeszensky obteve os títulos de Engenheiro Eletricista, Mestre em Engenharia Elétrica, Doutor em Engenharia Elétrica, Livre Docente e Professor Titular na área de Telecomunicações, pela Escola Politécnica da USP em 1972, 1981, 1989, 1992 e 2006, respectivamente. Iniciou sua carreira profissional, em 1973, na AEG-Telefunken do Brasil, na área de Telecomunicações. Em 1974 transferiu-se para a Fundação para o Desenvolvimento Tecnológico da Engenharia-FDTE onde, como gerente de projetos, coordenou mais de 30 projetos nas áreas de Telefonia Digital, Telemetria Hidrometeorológica, Supervisão de Sistemas em Tempo Real e áreas afins. Foi consultor autônomo da iniciativa privada, no período 1976/86, na área de desenvolvimento de aparelhos eletro-médicos. Autor do livro Sistemas Telefônicos (Editora Manole, 2004) e de cerca de 90 trabalhos técnicos/científicos, apresentados em Simpósios/ Congressos/revistas especializadas, nacionais e internacionais, nas áreas de atuação acima. Professor da EPUSP desde 1978, em dedicação exclusiva a partir de 1990, na área de Telecomunicações, respondendo pelas disciplinas: Comunicações Digitais II e Sistemas Telefônicos, em nível de graduação e Comunicação por Espalhamento Espectral (Spread Spectrum), Tópicos Especiais em Comunicações Digitais e Teoria e Aplicações das Sequiências de Código, em nível de pós-graduação. Coordenador do LCS-Laboratório de Comunicações e Sinais do Departamento de Engenharia Eletrônica da EPUSP, no período de 1995/97, 1999/2001 e a partir de 2003. Representante do PTCDepartamento de Engenharia de Telecomunicações e Controle na CG-Comissão de Graduação da EPUSP, desde 2003. Líder do Grupo de Pesquisa em Telecomunicações do Diretório de Grupos de Pesquisas no Brasil, do CNPq. General Co-Chairmen do ITS'98-SBT/IEEE International Telecommunications Symposium, realizado em SP de 9 a 13/8/1998. General Chairman do ISSSTA06, 2006 IEEE Ninth International Symposium on Spread Spectrum Techniques and Applications, realizado em Manaus de 28 a 31/08/2006. Assessor especial da TELESP-Celular para a implantação da digitalização na banda A de Telefonia Móvel Celular, em São Paulo, no período de 1997/98. Coordenador dos convênios de cooperação técnica entre a EPUSP e a Ericsson do Brasil, Motorola do Brasil e TELESP - Celular, desde 1999. Foi professor visitante da UPC-Universitat Politécnica de Catalunya, Barcelona-Espanha em 1995 e da TUB-Technical University of Budapest, Hungria em 2001. 